\title{
Reductive nanometric patterning of graphene oxide paper using electron beam lithography
}

Gil Gonçalves ${ }^{a *}$, Jérôme Borme ${ }^{b}$, Igor Bdkin ${ }^{a}$, Ankor González-Mayorga ${ }^{c}$, Gonzalo Irurueta $^{a}$, Helena I. S. Nogueira ${ }^{d}$, María C. Serrano ${ }^{e, f, g}$, Pedro Alpuim ${ }^{b, e, h}$ Paula A.A.P. Marques $^{a^{*}}$

a TEMA-NRD, Mechanical Engineering Department, University of Aveiro, 3810-193 Aveiro, Portugal

${ }^{\mathrm{b}}$ INL - International Iberian Nanotechnology Laboratory, 4715-330, Braga, Portugal

${ }^{c}$ Hospital Nacional de Parapléjicos (HNP), Servicio de Salud de Castilla-La Mancha (SESCAM), Finca La Peraleda s/n, 45071-Toledo, Spain

${ }^{\mathrm{d}}$ CICECO, Department of Chemistry, University of Aveiro, 3810-193 Aveiro, Portugal

e Instituto de Ciencia de Materiales de Madrid (ICMM), Consejo Superior de Investigaciones Científicas (CSIC), 28049-Madrid, Spain

${ }^{\mathrm{f}}$ Materials Science Factory, ICMM-CSIC, 28049-Madrid, Spain

g Joint Research Unit "Design and Development of Biomaterials for Neural Regeneration", HNP-SESCAM, Joint Research Unit with CSIC

${ }^{\mathrm{h}}$ CFUM-Center of Physics of the University of Minho, 4710-057, Braga, Portugal

*Corresponding authors.

E-mail: ggoncalves@ua.pt (Gil Goncalves);E-mail: paulam@ua.pt (Paula Marques) 


\section{Reductive nanometric patterning of graphene oxide paper using electron beam lithography}

Gil Gonçalves ${ }^{a *}$, Jérôme Borme ${ }^{b}$, Igor Bdkin ${ }^{a}$, Ankor González-Mayorga ${ }^{c}$, Gonzalo Irurueta $^{a}$, Helena I. S. Nogueira ${ }^{d}$, María C. Serrano ${ }^{e, f, g}$, Pedro Alpuim ${ }^{b, e}$ Paula A.A.P. Marques $^{a^{*}}$

a TEMA-NRD, Mechanical Engineering Department, University of Aveiro, 3810-193 Aveiro, Portugal

${ }^{\mathrm{b}}$ INL - International Iberian Nanotechnology Laboratory, 4715-330, Braga, Portugal

${ }^{c}$ Hospital Nacional de Parapléjicos (HNP), Servicio de Salud de Castilla-La Mancha (SESCAM), Finca La Peraleda s/n, 45071-Toledo, Spain

${ }^{\mathrm{d}}$ CICECO, Department of Chemistry, University of Aveiro, 3810-193 Aveiro, Portugal

e Instituto de Ciencia de Materiales de Madrid (ICMM), Consejo Superior de Investigaciones Científicas (CSIC), 28049-Madrid, Spain

${ }^{\mathrm{f}}$ Materials Science Factory, ICMM-CSIC, 28049-Madrid, Spain

g Joint Research Unit "Design and Development of Biomaterials for Neural Regeneration", HNP-SESCAM, Joint Research Unit with CSIC

${ }^{\mathrm{h}}$ CFUM-Center of Physics of the University of Minho, 4710-057, Braga, Portugal

*Corresponding authors. E-mail: ggoncalves@ua.pt (Gil Goncalves); paulam@ua.pt (Paula Marques)

Keywords: graphene oxide paper; reduction patterning; electron beam lithography; conductivity; nanoindentation; cytocompatibility 


\section{Abstract}

Electron beam lithography (EBL) was used for preparing nanostructured reduced patterns on the GO paper surface, while preserving its mechanical resistance and flexibility. Different EBL parameters, like dose and time of exposure for patterning were tested. SEM analysis showed the consequent increase of contrast of the reduced stripes on the patterned regions due to the increase of electron beam doses. Moreover, surface potential microscopy experiments also exhibited a clear contrast between the patterned and non-patterned regions. Structural analysis of the patterned paper through X-ray diffraction and nanoindentation showed that the interlayer distance between GO sheets decreases after reduction allowing the increase of the Hardness and Young modulus that makes this material able to be manipulated and integrated on different devices. Furthermore, we also observe that exposed areas to electron beam reduction process show an increase in the electrical conductivity up to $3 \times 10^{4}$ times. The developed flexible GO films can have interesting applications such as biosensors or templates for inducing tissue regeneration, by providing a surface with differently patterned cues with contrasting electron mobility. Preliminary in vitro studies with L929 fibroblasts support the cytocompatible nature of this patterned GO paper. 


\section{Introduction}

Nowadays, the wet chemical exfoliation of graphite is one of the most used methodologies to prepare graphene derivatives, with the advantage of large scale production, technological simplicity, high efficiency and low cost of the procedure.[1], [2]

Graphene oxide (GO) results when the exfoliation is done in aqueous oxidative medium, then retaining a high degree of oxidation, with a $\mathrm{C} / \mathrm{O}$ ratio achieving the maximum value of 2.5 , usually designated as the threshold limit [3]. This particular feature of GO provides it with a high hydrophilic character allowing the preparation of highly stable aqueous colloidal suspensions [4]. The chemical structure of GO is generally described by the model of Lerf-Klinowski that defines an atomic layer of carbon atoms in a hexagonal structure with hydroxyl and epoxy groups on the plane and carbonyl and carboxylic groups on the periphery [5]. Recently, Dimiev et al. proposed that the GO structure is dynamic in aqueous solution suggesting that $\mathrm{GO}$ is a metastable nanomaterial at room conditions [6]. Kim et al. observed that a quasi-equilibrium of GO structure was reached after a relaxation time of a month, resulting in a chemical structure rich in hydroxyl groups and devoid of epoxide groups [7]. The reduction of GO can be promoted through chemical [8] or thermal [9] treatments that remove most of the oxygen functional groups and allow the partial restauration of $\mathrm{sp}^{2}$ carbon bonds[10]. However, when compared to graphene, the quality standard of this graphene derivative is low, since it contains a high density of structural defects and a remaining percentage of oxygen functional groups.

However, the structural heterogeneity of GO can be an advantage for certain applications. In fact, the high versatility of GO arises from its chemical diversity based on the combination of aromatic and oxidized domains in a single atomic sheet. These 
characteristics allow its functionalization through several chemical and physical approaches using small molecules, nanoparticles or structures building-up new hybrid materials. Also, GO nanosheets have the ability to interact between them allowing their self-assembly into macroscopic structures like films or three dimensional microporous architectures, like foams $[11,12]$. The heterogeneity of GO chemical structure allows the establishment of hydrogen bonds between the oxygen functional groups or/and by the assembly of hydrophobic domains. A controlled assembly of the GO sheets through the interactions of functional groups in the basal planes leads to an ordered stacking, forming robust films [13]. However, if the interactions are preferentially achieved through the oxygen functional groups located at the edges of GO sheets, the creation of three-dimensional microporous architectures is favoured [14]. This new class of materials has been recently designated as solvated graphene, a new emerging area with enormous challenges and opportunities in multidisciplinary fields [15].

The first successful attempt to prepare GO films, usually designated as a GO paper, consisted on the simple flow-directed assembly of individual GO sheets [13]. The horizontally ordered assembly of solvated GO was guaranteed by the constant flow of the liquid from the suspension, which avoids aggregation allowing the individual sheets to reach an equilibrium between interconnecting forces (hydrogen bridges and $\pi-\pi$ stacking) and repulsive colloidal forces (hydration and electrostatic forces), at the filter surface. After drying, the resultant membranes showed high flexibility and stiffness. This approach is still commonly used for the preparation of GO paper nanocomposites with ordered structure and high performance for several applications [16-20]. Interestingly, a multilayer GO film is considered a metastable material at room conditions because it continuously undergoes chemical and structural changes, which result in a persistent decrease of the $\mathrm{C} / \mathrm{O}$ ratio [7]. The metastable state provides the 
opportunity to enhance the transformation of several functional groups by applying soft physical treatments, such as low temperature treatment processes [21], in order to modulate the final properties of GO. On the other hand, when carbon nanomaterials are exposed to electrons or ions, their microstructure evolves through the creation of defects [22]. Krashnenniniko et al. reported how these effects can be used to tailor the properties of carbon nanomaterials $[23,24]$. In the case of $\mathrm{GO}$, exposure to direct sunlight radiation leads to the elimination of oxygen in the form of water vapour [25].

The patterning based on reduction of GO has been obtained in the literature by a variety of techniques. The existing reports are collected in table X. Several techniques are based on local heating. It is assumed that thermal reduction starts occurring in a range from $100{ }^{\circ} \mathrm{C}[26]$ to $180{ }^{\circ} \mathrm{C}[27]$, and it is considered that $200-500{ }^{\circ} \mathrm{C}$ is an effective temperature range leading to reduction $[28,29]$ by elimination of carboxyl groups.[30] Higher local temperatures lead to ablation [29]. The use of a scanning focused laser light is a common way to obtain local heating, as GO is a light absorber, absorbing $63 \%$ of incident light within $1 \mu \mathrm{m}[31]$ in average over the visible range, which leads to a rapid decrease of temperature along the depth. This decrease will be steeper for shorter wavelengths [32], leading to different patterning performance with different wavelengths used in thicker films and papers. Besides direct scanning, other patterning strategies include shadow masks, used with either photographic flash[31,33] or laser irradiation [34], and also the use of interference patterns [35-37]. Some authors combine several techniques to achieve patterns, such as laser ablation and chemical reduction [28], or pulsed laser deposition followed by thermal annealing [38]. Another thermallybased technique was the use of a heated AFM probe, leading to an extremely small lateral resolution of $12 \mathrm{~nm}$ [26]. Besides thermal processes, a photochemical reduction of GO [39] and other carbon materials can occur where water dissociation provides 
protons which bind to oxygen-rich functional groups, leading to their removal from the carbon backbone [40]. This phenomenon occurs for photon energies above a threshold of $3.2 \mathrm{eV}[30]$ and up to $300.0 \mathrm{eV}[39]$, while using higher energy laser lines caused the breaking of carbon-carbon bonds [40]. GO surface patterning was also performed using an extreme-UV (EUV) radiation source showing high efficiency on the reduction process, with very high resolution (nanometre range) and absence of photothermal effects [41]. The same authors showed that GO photoreduction patterning could be significantly improved with the use of high-performance light sources like vacuum UV (VUV) synchrotron radiation [42]. In parallel, others reported the reductive effect of electron beams, [43] and the lithographic writing of reduced patterns was reported using a $20 \mathrm{keV}$ electron beam [32]. More recently, devices made of GO with micrometer scale reduced patterns have been produced using electron exposure at $25 \mathrm{keV}$ [44]. Furthermore, it was observed that electron irradiation at $10 \mathrm{MeV}$ can induce the chemical bonding between the adjacent reduced GO sheets by $\mathrm{sp}^{3}$ carbon formation, increasing the mechanical hardness and electrical conductivity of the paper [45].

In the context of biomedical applications, EBL has been explored as an attractive technique for the fabrication of submicron (down to the nanoscale) topographies on a diverse palette of materials, including protein patterns for multiplexed cytokine detection,[46] substrates with grooved topographies to modulate cell differentiation, [47] and membranes for in vitro models of barrier tissue [48]. However, its application to graphene-based materials has been scarcely investigated, despite the tremendous biological interest of these materials due to their attractive physico-chemical properties and versatile nature for functionalization. For example, graphene-based materials are under study to be used as part of engineered devices to promote cell growth and alignment [49], to interface native tissues [50], and to modulate cell differentiation 
processes [51], which could benefit from the application of versatile patterning techniques, such as EBL. Although the compatibility of graphene based materials in contact with biological systems remains a matter of open debate [52], published results to date support the existence of a safe range of conditions in which these materials display biocompatible behaviour and do not initiate any toxic responses [53].

The majority of the studies previously mentioned on the patterning of GO, focused on GO thin films obtained by spin-coating on hard substrates, scarce work has been done on self-sustaining GO paper. Herein, we report the development of self-stained GO paper where direct-write EBL was used to create micrometer-scale conductive lines, using a $100 \mathrm{keV}$ electron beam. To the best of our knowledge no studies were reported about GO reduction in the energy range used in this study, previous works used $20 \mathrm{keV}$ [32] and $25 \mathrm{keV}$ [44] for $\mathrm{GO}$ films, and $5 \mathrm{MeV}$ [54] and $10 \mathrm{MeV}$ [45] for GO paper. The GO paper prepared by self-assembly of the GO sheets was reduced according to a free pattern chosen to be a series of parallel lines, using different electron doses and beam step sizes in order to effectively obtain a reduced pattern at the GO paper surface. A discussion of the characterization results of the GO patterned paper obtained for the different working conditions is presented. Finally, in order to elucidate the biocompatible nature of the patterned GO paper for future biological uses, we carried out cytocompatibility tests with murine L929 fibroblasts. Specifically, cell adhesion, growth and morphology were investigated both on patterned and non-patterned areas of the GO paper by confocal laser scanning microscopy (CLSM) and SEM.

\section{Experimental}

\subsection{Material and Methods}

\subsubsection{Material}


GO was supplied by Graphenea with a concentration of $4 \mathrm{mg} / \mathrm{mL}$. The GO was repeatedly washed with ultrapure water by centrifugation until reaching neutral $\mathrm{pH}$. Chemical reagents and antibodies were purchased from Sigma-Aldrich and used as received, unless otherwise indicated. Cell culture media components were obtained from Lonza.

\subsubsection{GO paper preparation}

The preparation of the GO paper was based on the method described by Dikin et al. [13] Briefly it consists of the filtration of $20 \mathrm{~mL}$ of $\mathrm{GO}$ aqueous colloidal solution (3 $\mathrm{mg} / \mathrm{mL}$ ) over a Durapore membrane filter with diameter of $47 \mathrm{~mm}$ and pore size of 0.22 $\mu \mathrm{m}$, until complete drying. During filtration, the GO sheets tend to align at the interface with the filter and assembly horizontally forming a thin paper that can be easily removed after drying. The thickness of the final GO papers is dependent on the concentration and volume of the solution used, which can be tuned.

\subsubsection{GO paper patterning by electron beam lithography}

The GO paper patterning was performed using EBL (Vistec EBPG 5200) operated at $100 \mathrm{kV}$. The sample plane was set using a three-point alignment system. In order to account for the dilatation of the sample, which we estimate to be $\sim 100 \mu \mathrm{m}$, the base plane of the sample was set at $50 \mu \mathrm{m}$ below focus point, so that at all times during exposure the sample is placed within $\pm 50 \mu \mathrm{m}$ of the electron focus plane. The five experimental conditions tested for the patterning of GO paper are summarized on Table 1.

Table 1- Experimental conditions tested for the patterning of the GO paper by EBL. 


\section{ACCEPTED MANUSCRIPT}

\begin{tabular}{llllll}
\hline Exposure & $\mathbf{1}$ & $\mathbf{2}$ & $\mathbf{3}$ & $\mathbf{4}$ & $\mathbf{5}$ \\
\hline Dose $\left(\times 10^{3} \mu \mathrm{C} / \mathrm{cm}^{2}\right)$ & 0.8 & 8 & 80 & 800 & 8000 \\
Line length $(\mu \mathrm{m})$ & 600 & 600 & 600 & 600 & $300\left(^{*}\right)$ \\
Step size $(\mathrm{nm})$ & 25 & 20 & 20 & 20 & 20 \\
Dwell time $(\mathrm{ns})$ & 30 & 192 & 1919 & 19190 & 191904 \\
Beam linear velocity $(\mu \mathrm{m} / \mathrm{s})$ & 834 & 104 & 10.4 & 1.04 & 0.104 \\
& 53.73 & 104.78 & 617.01 & 5792.34 & 28776.55 \\
Exposure duration $(\mathrm{s})$ & $\approx 1 \mathrm{~min}$ & $\approx 1 \mathrm{~min} 45$ & $\approx 10 \mathrm{~min}$ & $\approx 1 \mathrm{~h} 35$ & $\approx 8 \mathrm{~h}$ \\
& & & & & \\
\hline
\end{tabular}

$\left(^{*}\right)$ half the length to reduce exposure time to reasonable value

\subsubsection{Materials Characterization}

SEM was performed using a FEI Nova NanoSEM 650. Electron acceleration was set to $2 \mathrm{kV}$ or $3 \mathrm{kV}$ and the images were acquired in secondary electron mode. Elemental analysis was performed using elemental-dispersion X-ray spectroscopy tool included in the SEM, with acceleration voltage of $7 \mathrm{kV}$.

Atomic Force Microscopy (AFM) and Kelvin Probe Force Microscopy were performed by Veeco AFM Multimode Nanoscope (IV) MMAFM-2. The conductive probes used were made of $\mathrm{Pt} / \mathrm{Ir}$ coated $\mathrm{Si}$ cantilever from Nanosensors; with a nominal force constant $15 \mathrm{~N} / \mathrm{m}$. The powder X-ray diffraction (XRD) patterns of GO paper were collected at room temperature in a continuous scanning mode (step $0.02^{\circ}$ and time $20 \mathrm{~s}$ ) on a Siemens D500 diffractometer with a secondary monochromator $\mathrm{CuK} \alpha \mathrm{X}$-radiation in the range $2 \theta=3-155^{\circ}$. X-ray photoelectron spectroscopy (XPS) spectra were acquired in an Ultra High Vacuum (UHV) system with a base pressure of $2 \times 10^{-10}$ mbar. The system is equipped with a hemispherical electron energy analyser (SPECS Phoibos 150), a delay-line detector and a monochromatic AlKa (1486.74 eV) X-ray source. High resolution spectra were recorded at normal emission take-off angle and with a passenergy of $20 \mathrm{eV}$. Fourier transform infrared spectroscopy (FTIR) spectra were recorded 
using a Bruker optics tensor 27 spectrometer coupled to a horizontal attenuated total reflectance (ATR) cell using 256 scans at a resolution of $4 \mathrm{~cm}^{-1}$. The samples were examined directly and data were obtained in transmittance mode. Nanoindentation measurements were performed using a three-sided pyramidal Berkovich diamond indenter having a nominal edge radius of $20 \mathrm{~nm}$ (faces 65.3 from vertical axis) attached to a fully calibrated nanoindenter (TTX-NHT, CSM Instruments).

Raman imaging was performed in a combined Raman-AFM-SNOM confocal microscope WITec alpha300 RAS+. A frequency doubled Nd:YAG laser operating at $532 \mathrm{~nm}$ was used as an excitation source with the power of $2 \mathrm{~mW}$ at the sample. Raman imaging experiments were performed by raster-scanning the laser beam over the samples and accumulating a full Raman spectrum at each pixel. Raman images were constructed by integrating over specific Raman bands using WITec software for data evaluation and processing.

\subsubsection{Cytocompatibility studies}

Prior to cell culture, GO paper samples were sterilized under UV radiation (30 min per side), placed in sterile standard 24-well polystyrene plates and pre-conditioned in culture media for $72 \mathrm{~h}$. Murine L929 fibroblasts were then seeded on the samples at a density of $2.0 \cdot 10^{4}$ cells per well in Dulbecco's Modified Eagle's Medium supplemented with $10 \%$ fetal bovine serum, streptomycin $\left(100 \mathrm{UI} \mathrm{ml}^{-1}\right)$, penicillin $\left(100 \mathrm{UI} \mathrm{ml}^{-1}\right)$, and L-glutamine $\left(1 \mathrm{mmol}^{-3}\right)$ (culture media). Cultured samples were maintained in a sterile incubator at $37{ }^{\circ} \mathrm{C}$ in a $\mathrm{CO}_{2}$ atmosphere $(5 \%)$ for $96 \mathrm{~h}$.

For adhesion and growth studies by CLSM, samples were fixed with paraformaldehyde (4\% in phosphate buffer saline, PBS) at room temperature for $12 \mathrm{~min}$ and then incubated with a specific antibody against vimentin, a common protein 
constituent of cytoskeleton in most cells. The secondary antibody used was Alexa Fluor® 594 IgG $(\mathrm{H}+\mathrm{L})$ (Life technologies). Both primary and secondary antibodies were dissolved in PBS containing saponin $(0.25 \%)$ and fetal serum $(2 \%)$ to guarantee cell permeability and to block any non-specific bindings, respectively. Each antibody was incubated for $1 \mathrm{~h}$ at room temperature in darkness. Cell nuclei were labelled with

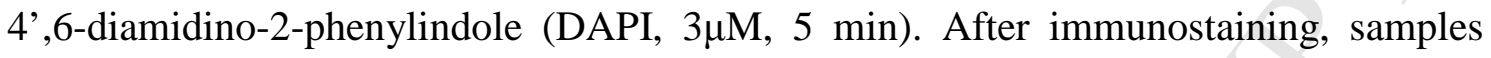
were visualized by using a Leica TCS SP5 microscope. Alexa Fluor ${ }^{\circledR} 594$ was excited at $594 \mathrm{~nm}$ with a helium-neon laser and detected in the range $625-689 \mathrm{~nm}$, while DAPI excitation was carried out at $405 \mathrm{~nm}$ with a diode UV laser and its detection in the range 423-476 $\mathrm{nm}$. Images in reflection mode were recorded at $488 \mathrm{~nm}$ to visualize the substrate surface and better locate cells with respect to the patterns.

For morphological studies by SEM, cultured samples were rinsed in PBS twice and fixed with glutaraldehyde $(2.5 \%$ in PBS) for $45 \mathrm{~min}$. After washing in distilled water, dehydration was performed by using series of ethanol solutions for $15 \min (2$ washes) and a final dehydration in absolute ethanol for $30 \mathrm{~min}$. Samples were then dried at room temperature for at least $24 \mathrm{~h}$. After mounting in stubs and gold coating under vacuum, the morphology of the samples was characterized by using a Hitachi S-4700 microscope.

\section{Results and Discussion}

\subsection{Reducing patterning of GO paper by electron beam lithography}

The reducing patterning of GO paper was performed under increasing EBL doses using an apparatus as can be visualized in Figure 1a). Five identical patterns with 999 lines of $200 \mathrm{~nm}$ width and $1 \mu \mathrm{m}$ centre-to-centre pitch were made. The five patterns were performed with doses successively higher, increasing in factors of 10, starting with the 


\section{ACCEPTED MANUSCRIPT}

dose normally used for polymethylmethacrylate (PMMA) exposures $\left(800 \mu \mathrm{C} / \mathrm{cm}^{2}\right)$ and up to $8 \mathrm{C} / \mathrm{cm}^{2}$. The complete exposure parameters are shown in Table 1 . After exposure, all samples, with exception of the one subjected to the lowest dose $\left(800 \mu \mathrm{C} / \mathrm{cm}^{2}\right)$ showed visible morphological modifications, in particular, a swelling of the paper extending to several hundreds of micrometres around the exposed area (Fig. 1b) and c)).

a)

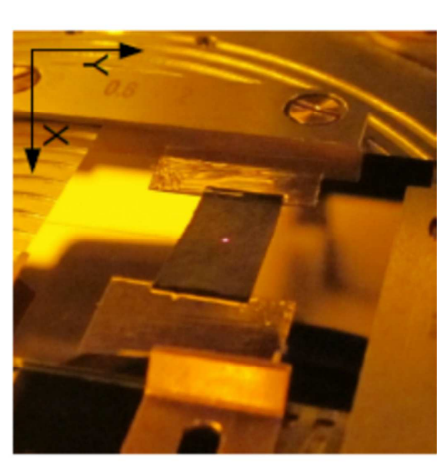

b)
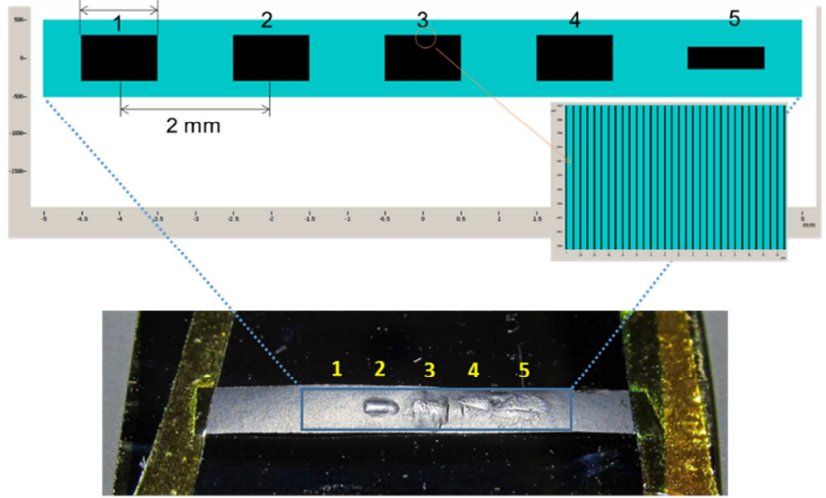

c)

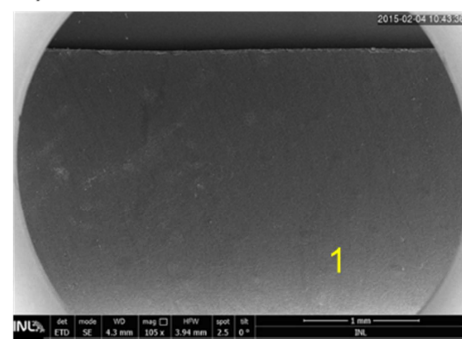

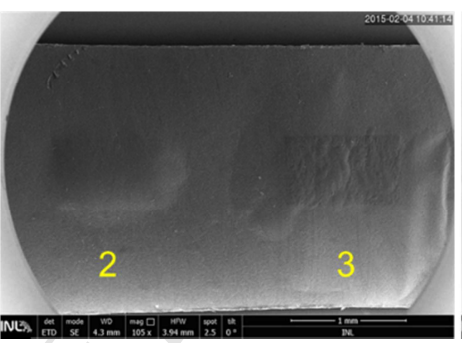

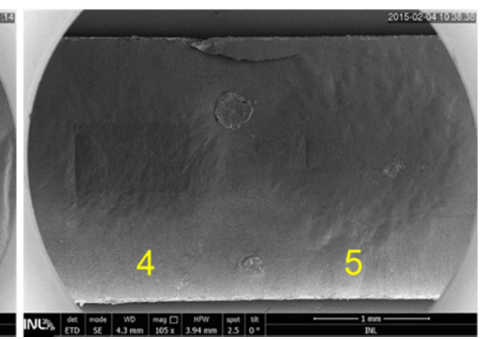

Figure 1. a) Photograph of a detail of the EBL apparatus used for the surface reduction patterning of GO paper. b) Schematic representation and optical photograph of the five exposed areas in a strip of the GO paper sample. c) Low-magnification SEM micrographs of the exposed samples, showing morphological changes in and around the rectangular exposed area, for the two largest doses used (4 and 5).

As a preliminary characterization, the exposed areas were observed by optical microscopy and SEM. Regarding the SEM analysis a comment should be firstly introduced: because electron beam was used for both creating the effect and observing it, this raises the question of the interference of observation conditions with the observed object. Indeed it has been reported that graphene materials can be altered by 
electron beams in the range $5 \mathrm{keV}$ to $20 \mathrm{keV}[55,56]$. Cutting through carbon nanotubes has also been reported at $1 \mathrm{keV}$, however it required significant exposure time with the beam focused on the cut line and the process was strongly accelerated by the presence of water in the atmosphere [57]. Due to the small magnifications, the much shorter time required for the samples imaging compared to the large exposure times required to produce them, we assume that the observation did not lead to significant changes in the material. Also, any artefact coming from the SEM view from the top would uniformly affect the image, while the effect to be detected follows a known pattern. Finally, at an energy of 2 to $3 \mathrm{keV}$, the electron contrast observed in the images is known to come from the surface of the sample, therefore any potentially reducing effect would occur in that region, while the effect of the $100 \mathrm{keV}$ electron exposure is expected to extend to the full thickness of the material, so any effects of electron microscopy would be easy to differentiate from those arising from EBL.

Optical observations showed that morphological changes were present in samples exposed to electron doses equal or higher to $8000 \mu \mathrm{C} / \mathrm{cm}^{2}$. Highly magnified images of regions 4 and 5 showed a faint contrast in the shape of the exposed stripes (Figure 2) as was also reported by others [30], which we analysed as a peak in the power spectrum density of the SEM images at the expected spatial frequency for these two regions (Figure S1 - supplementary information). We ascribe this different contrast to a different conductivity of the GO paper after exposure to the electron beam.

The dose used in area 5 is nearly the maximum possible to attain with the electron lithography tool, so images 2-5 in Figure 2 shows the largest effect that a focused 100 $\mathrm{keV}$ electron exposure can practically cause to the structure of GO paper. 

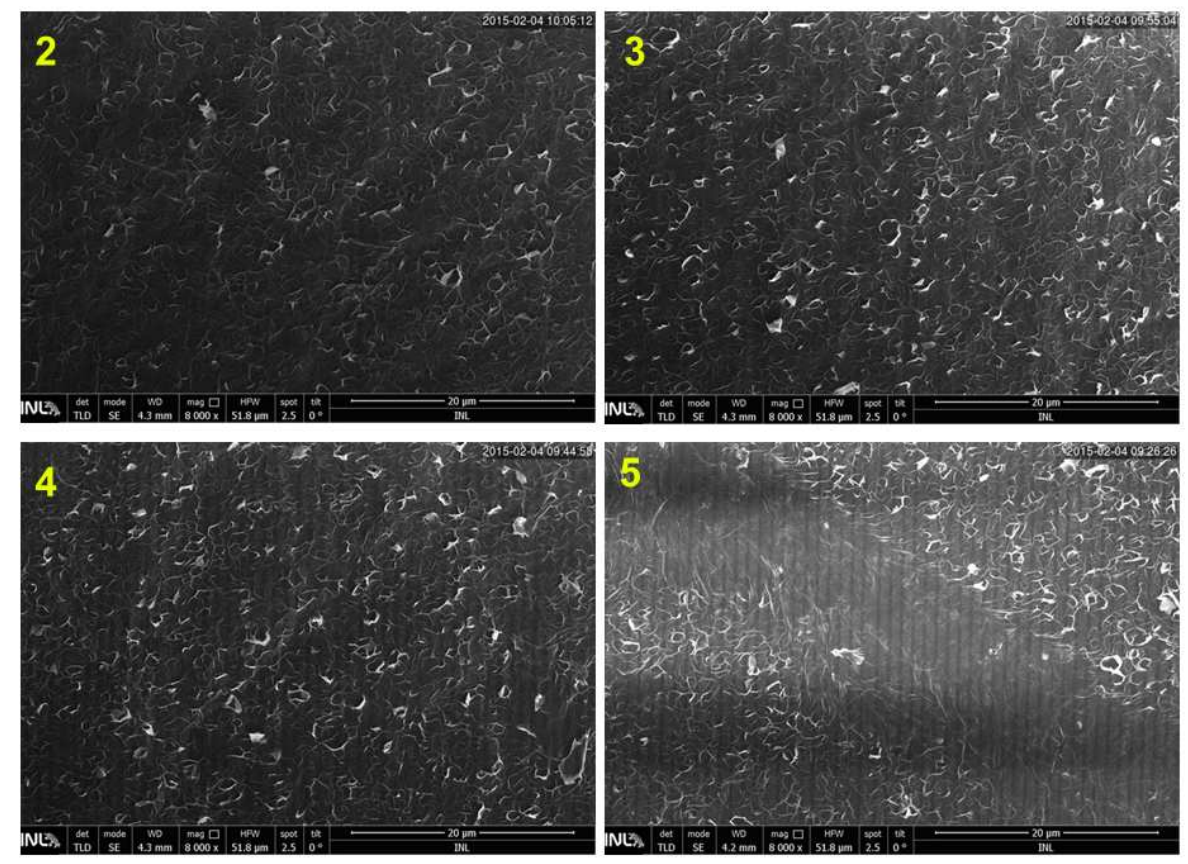

Figure 2. High magnification SEM micrographs of the areas exposed to different doses (2, 3, 4 and 5). For depositions at conditions 4 and 5, with the highest dose, a stripe pattern is visible.

In order to better understand the depth and lateral reach of the EBL, a computer simulation of the electron scattering process resulting from the interaction with the sample was performed. Figure 3 shows the depth of penetration of energy deposited by the electrons, as a function of the incident electron energy. The simulation was performed using Casino 2.48 [58], assuming a ratio of carbon to oxygen $\mathrm{C} / \mathrm{O}=2.7$. For each voltage, the distribution of energy by position was calculated in a $1 \times 1 \times 1000$ grid $(\mathrm{x} \times \mathrm{y} \times \mathrm{z})$. The $50 \%$ and $90 \%$ quantiles were extracted from a cumulative distribution. The latter appears to increase rapidly with the energy $\mathrm{E}$ of the incident electrons, following $\mathrm{E}^{1.75}$. 


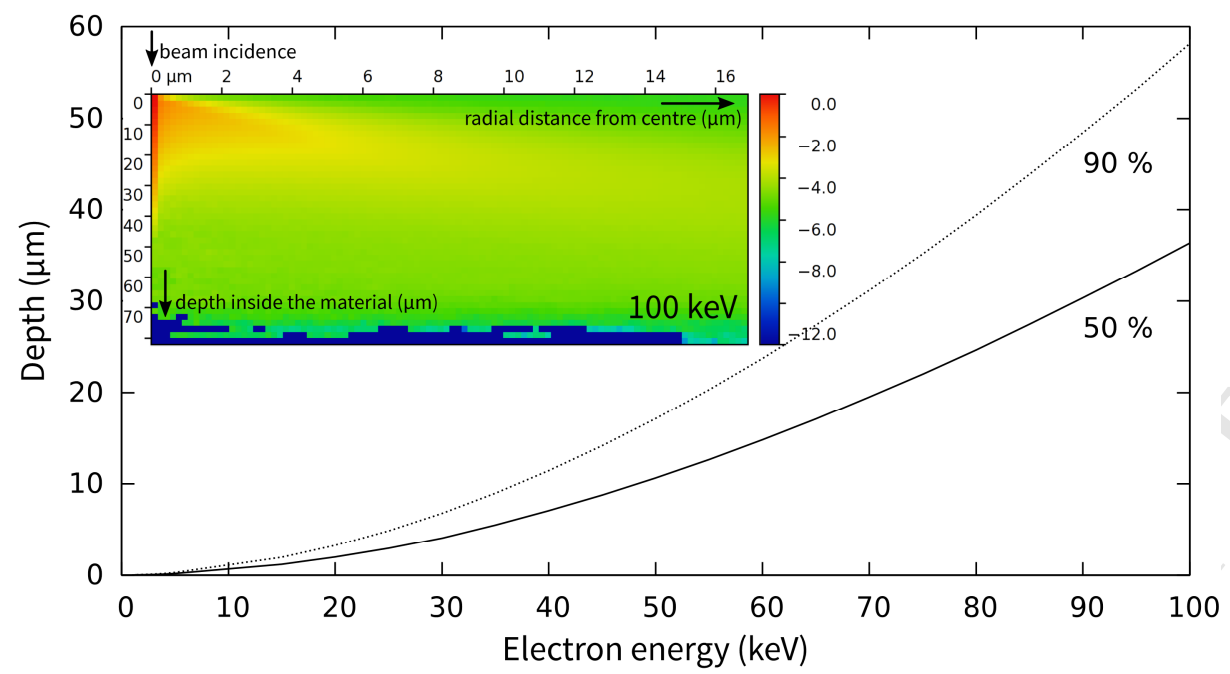

Figure 3. Depth of penetration of energy transferred by the electrons onto a GO material modelled as a material containing carbon and oxygen with $\mathrm{C} / \mathrm{O}=2.7$, simulated with Casino 2.48 [58], as function of the acceleration voltage of the incident electrons. In inset, the cross-sectional distribution of deposited energy at $100 \mathrm{kV}$, plotted as a radial distribution. The arrow at $(0,0)$ in the inset indicates the place of beam incidence. The colour scale corresponds to the decimal logarithm of energy distribution.

According to these results, for experiments performed in the range of $20 \mathrm{keV}$ available in the literature $[32,44]$, the depth of energy penetration is about $2 \mu \mathrm{m}$, which is adequate for the spin-coated thin films these authors used. With the incident energy of $100 \mathrm{keV}$ used in the present work, the depth within which half of the energy is deposited is calculated to be $36 \mu \mathrm{m}$, a penetration depth similar to the thickness of the self-standing paper itself. The inset in Figure 3 shows the spatial distribution of the deposited energy inside a cross section of a simulated GO paper. It was simulated using a $1000 \times 1000 \times 100$ cartesian grid in Casino and further processed to extract the radial distribution. The incident electron beam is located at the top left corner in the figure, and travels through the material along the vertical axis from top to bottom. The radial 
distance from the point of incidence is represented at the horizontal axis. According to the simulation, most of the energy is concentrated very close to the vertical axis, even at large depth. This means that the electron beam maintains directionality while being scattered in the material. The energy deposited at lateral distances of a few micrometres from the point of incidence is only a very small fraction of the total energy. The results of the simulation allow to conclude that the reduction is well defined laterally at the scale of the pattern used in this work. It occurs in nearly the whole thickness of the GO paper, with no need to resort to a particle accelerator facility like in previous work for samples of similar composition and thickness [45, 54].

The energy deposited by the electron beam is confined to the vicinity of the electron beam diameter; a small part of this energy is deposited within a radius of a few micrometers, between 10 and $20 \mu \mathrm{m}$ (Figure 3, inset), possibly leading to a partial reduction effect in a range around the desired pattern, compatible with the proximity effect observed by others[59]. The electron collisions with graphitic structures cause the creation and accumulation of defects, which is called the Wigner effect.[60] Upon thermal annealing, lattice defects reorganise in $\mathrm{sp}^{2}$ carbon bonds, approaching graphene structure. In GO, the temperature threshold for this effect has been shown to be lower than that in graphite, starting to occur near $100{ }^{\circ} \mathrm{C} .[45]$ In the present work, the beam scans the sample in vacuum $(5 \times 10-7$ mbar $)$ at very slow linear velocity, down to 0.1

$\mu \mathrm{m} / \mathrm{s}$ (Table 1), with the beam moving first along the short direction of the stripes. These conditions favour local heating and therefore the process of Wigner release. According to Prezioso et al.,[33] a decrease of 20 percentage points in the $\mathrm{C}-\mathrm{O}$ binding content corresponds to a minimum temperature of $50{ }^{\circ} \mathrm{C}$ locally during the exposure.

\subsection{Structural characterization of patterned GO paper}


The structural analysis of the GO patterned paper was performed on samples obtained under the conditions established for exposure number 5. However, in order to facilitate characterization with conductive AFM and Raman spectroscopy, the width of the exposed stripes was set to $2 \mu \mathrm{m}$ instead of $200 \mathrm{~nm}$. The patterned region contained 55 lines measuring $2 \mu \mathrm{m} \times 600 \mu \mathrm{m}$, spaced by $10 \mu \mathrm{m}$, as depicted in Figure 4 . The dose used was $10000 \times 10^{3} \mu \mathrm{C} / \mathrm{cm}^{2}\left(6.2 \times 10^{19} \mathrm{e}^{-/ \mathrm{cm}^{2}}\right)$ with a step size of $1 \mathrm{~nm}$.

At a large magnification, as shown in Figure $4 \mathrm{~b}$, there is a visible deformation of the GO paper within and around the area where it was exposed to the electron beam. Because of the changed visual aspect, one would expect changes in the local topography. However, studies of the topography by AFM did not show topographical contrast between the lines exposed to the electron beam and the surrounding areas (Figure 4). Such contrast would be expected as previous works using laser reduction have reported a thinning of the sample in the exposed area due to a more compact form of the reduced material [24]. However, in the literature, the thinning effect was not clearly visible in the topographic data in the case of electron exposure [44]. In this work, samples differ from others published, in that they have a much higher thickness than, e.g. the spin-coated films previously reported. The electron beam exposure of this sample resulted in large deformation and local fracture, which can be attributed to the pressure exerted by gases released during the reduction, at GO interlayers [41]. 
a)

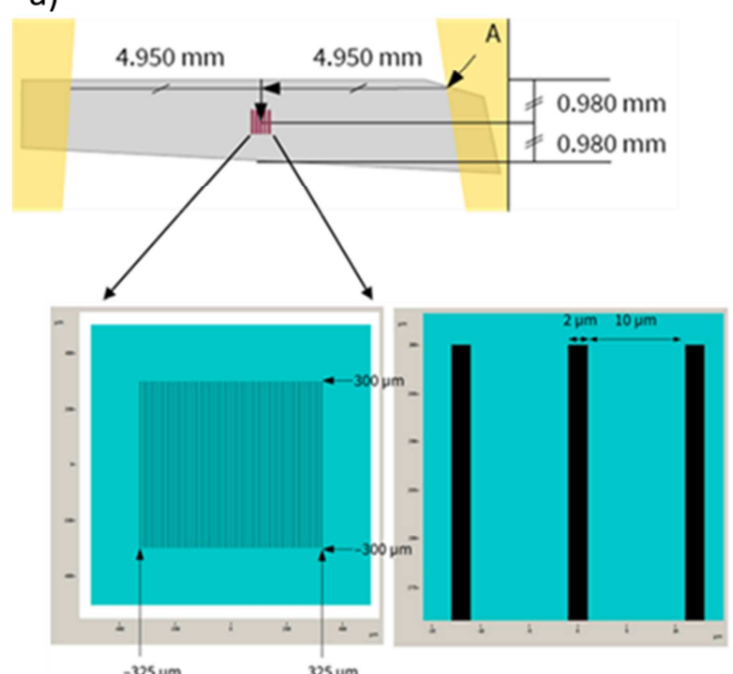

b)
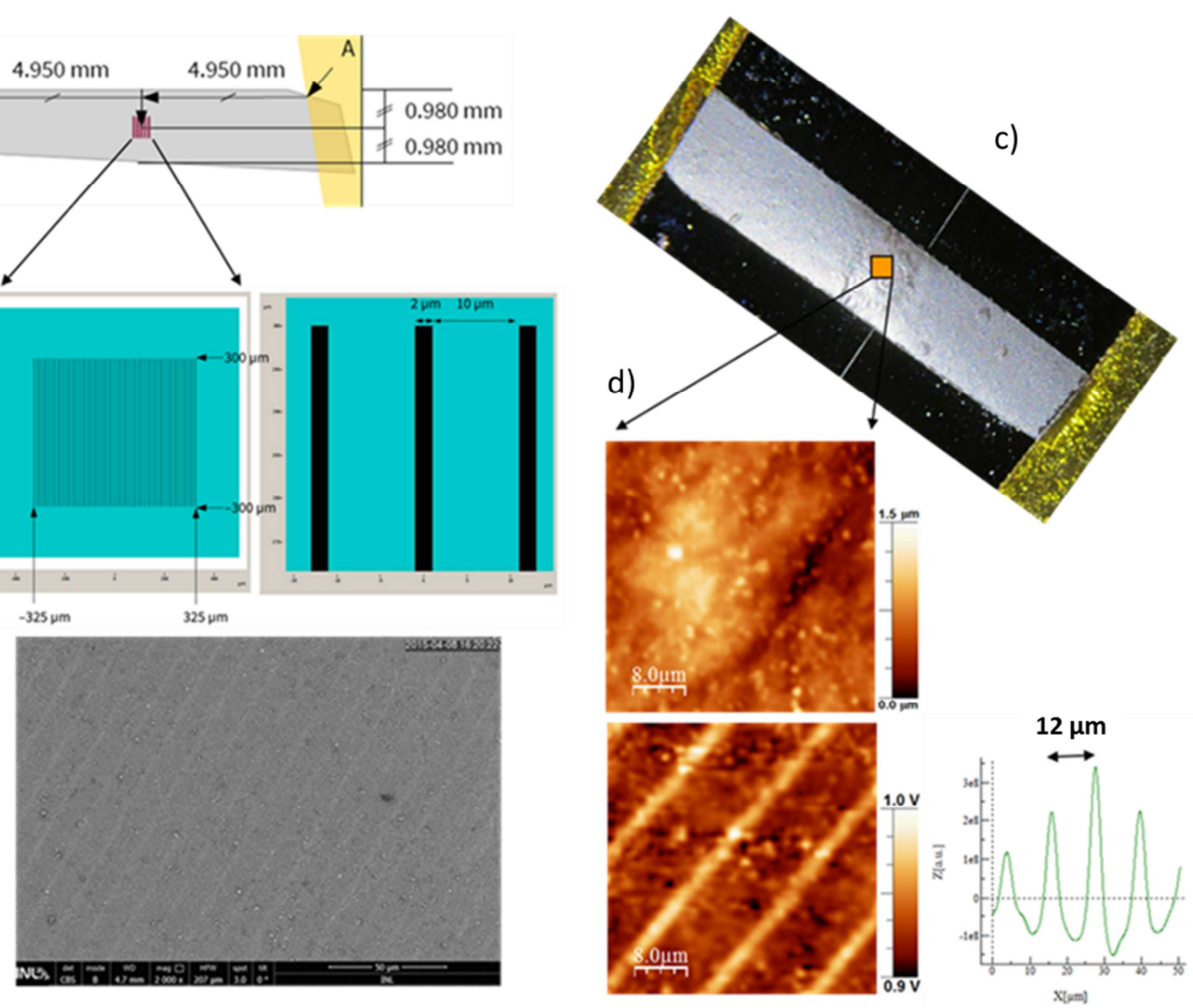

Figure 4. a) Schematic representation of the patterned performed on the surface of the GO paper by EBL. b) SEM image of patterned GO paper. c) Optical photograph of the exposed area on a strip of the GO paper sample. d) AFM measurements of the surface potential of the patterned GO paper, showing the predefined center-to-center distance between the reduced lines of $12 \mu \mathrm{m}$.

In order to make evident the difference between the exposed and unexposed areas in scanning probe microscopy, measurements of surface potential were performed using a silicon tip coated with Pt $40 \mathrm{~nm}$. These measurements showed a difference of $0.1 \mathrm{eV}$ in surface potential between the exposed and unexposed areas at a period of $12 \mu \mathrm{m}$, corresponding to the exposed pattern (Figure $4 \mathrm{~d}$ )). This is compatible with a change in the oxygen composition of the surface, as it has been calculated that the nature and organization of oxygen groups at the surface of the $\mathrm{rGO}$ areas can lead up to $2.5 \mathrm{eV}$ 


\section{ACCEPTED MANUSCRIPT}

change in the work function [61]. Lateral force microscopy showed no significant changes in adhesion force between patterned and unpatterned regions, which suggest that the carbon structure was not altered by the EBL exposure (Figure S2 supplementary information).

One sample was exposed to the electron beam from edge to edge, across its full width, in order to observe the exposed area formed at the edge in the SEM image of the crosssection (Figure 5a)). A closer look at the cross-section (Figure 5b) shows the expansion of GO sheets in the paper, which nearly doubles the thickness, increasing from about 40 $\mu \mathrm{m}$ in the unexposed bulk material to about $70 \mu \mathrm{m}$ around the exposed area, for an extension that spans more than $300 \mu \mathrm{m}$ to each side of the exposed line. In this region, a high number of cracks and voids are visible, aligned parallel to the surface of the film.

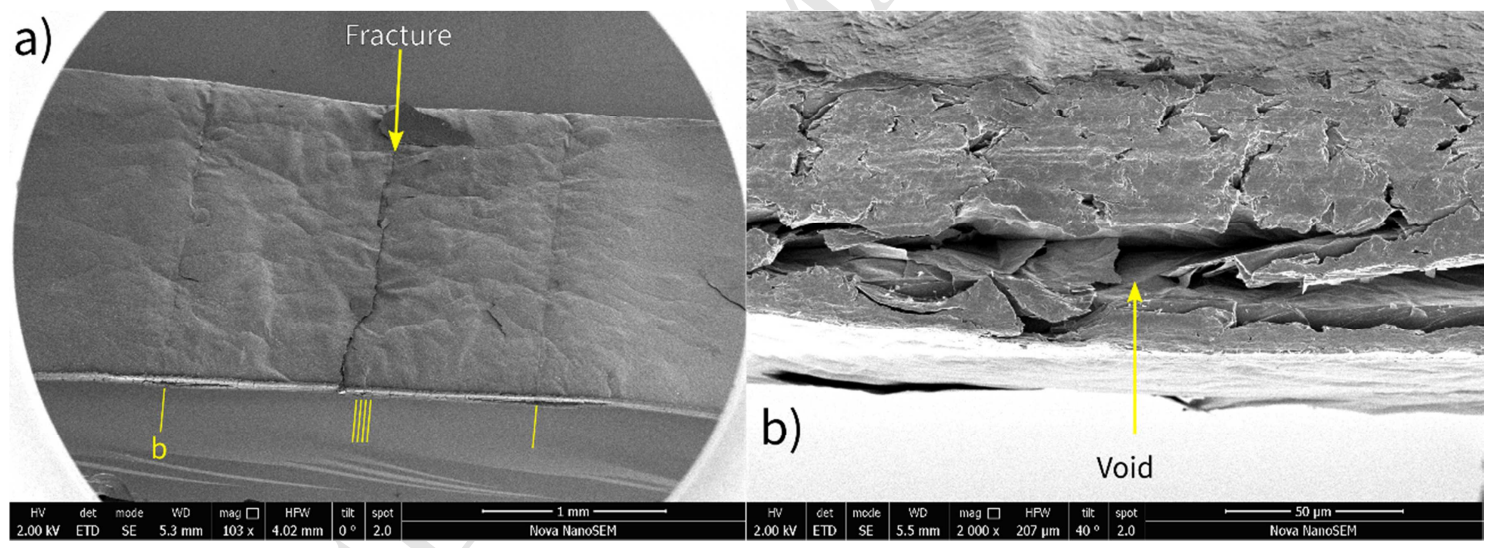

Figure 5. SEM observation of a sample patterned using the $100 \mathrm{kV}$ electron beam in the shape of lines crossing its entire width. a) Low magnification image under a $45^{\circ}$ tilt. The marked lines denote the places of exposure: one central region with eight exposed lines, and two single lines on either side of the central region. The central area exhibits a continuous fracture across the sample, which appeared during the manipulation of the sample. b) Close-up on the area marked b in image a), under a tilt of $85^{\circ}$. This image shows the structure in sheets of GO and the voids caused by reduction, following the 
exposure. It also allows evaluation of the thickness of the GO paper in the area exposed to the electron beam.

The preparation of GO paper by randomly stacked and densely packed GO sheets on top of the filter, makes this material prone to the intercalation of water molecules [62]. The reduction process by electron beam irradiation promotes the gradual increase in concentration of gases and water vapour that exert high pressure to the surrounding matter up to a critical point, when the van der Waals forces between adjacent GO sheets are overcome. An expansion in the planar direction is helped by the lamellar structure and high flexibility of the GO sheets, which allows for the observed deformation. McAlliste et al. also reported a thermal expansion mechanism of GO, attributed to the increased interlayer pressure by thermal decomposition of hydroxyl and epoxy groups, in the syntheses of functionalized graphene nanosheets [63].

The areas of the GO paper exposed to the electron beam also become more brittle. Besides the cracks, which originate and propagate following sites of stress concentration, a contributing factor specific to GO is that the diminished oxygen content in the reduced area brings the material properties back in the direction of those of graphite. An illustration of the brittle nature of the exposed area is shown on Figure 5a, where the sample was fractured along the direction of the exposed lines after simple manual manipulation leading to sample bending. This result is compatible with the recent report [64] where unconstrained fast thermal reduction of GO films at $1000{ }^{\circ} \mathrm{C}$ results in the formation of brittle and cracked films, in contrast to the constrained reduction that allows to obtain robust and flexible films due to the improved graphitization of the structure promoted by the applied pressure.

The cross-section observed in Figure $5 \mathrm{~b}$ is coherent with the results obtained by AFM where no change in height could be observed within the patterned area. Indeed, the 
deformation provoked by the exposure, of the same order as the GO paper thickness, reaches hundreds of micrometres laterally. Because of this lateral distance, largely exceeding the spatial period of the reduced lines, the much smaller thickness difference between exposed lines and unexposed surrounding areas is hidden.

$\mathrm{XRD}$ on the patterned and non-patterned regions of the GO papers allows obtaining further information about the structural changes observed after EBL exposure (Figure 6). GO paper diffraction peak is observed at $2 \theta=10.15^{\circ}$, which corresponds to the interlayer separation $\mathrm{d} \approx 8.702 \AA$ (Figure 6a). It is interesting to note that the GO peak is not broad $\left(\Delta 2 \theta=0.96^{\circ}\right)$, which means that the layered structure of the GO is preserved with thickness of $\approx 12.3 \mathrm{~nm}$. Two different peaks are observed after the deconvolution of XRD pattern for exposed GO paper, the first one with the same correspondence of non-exposed paper at $2 \theta=10.20^{\circ}$, with interlayer separation of $\mathrm{d} \approx 8.749 \AA$, and a second one at $2 \theta=11.53^{\circ}$ corresponding to an interlayer separation of $d \approx 7.673 \AA$, with a peak width of $\Delta 2 \theta=1.549^{\circ}$, showing a preserved ordered state with a thickness of $19.8 \mathrm{~nm}$. Those results show that in fact the EBL is able to reduce the GO sheets and decrease the interplanar distance as schematized in Figure 6b). Recently, it was reported that the presence of the XRD peaks at the higher angles for thermally obtained rGO paper, indicates the presence of numerous domains of densely stacked/overlapping layers of rGO [9]. 
b)

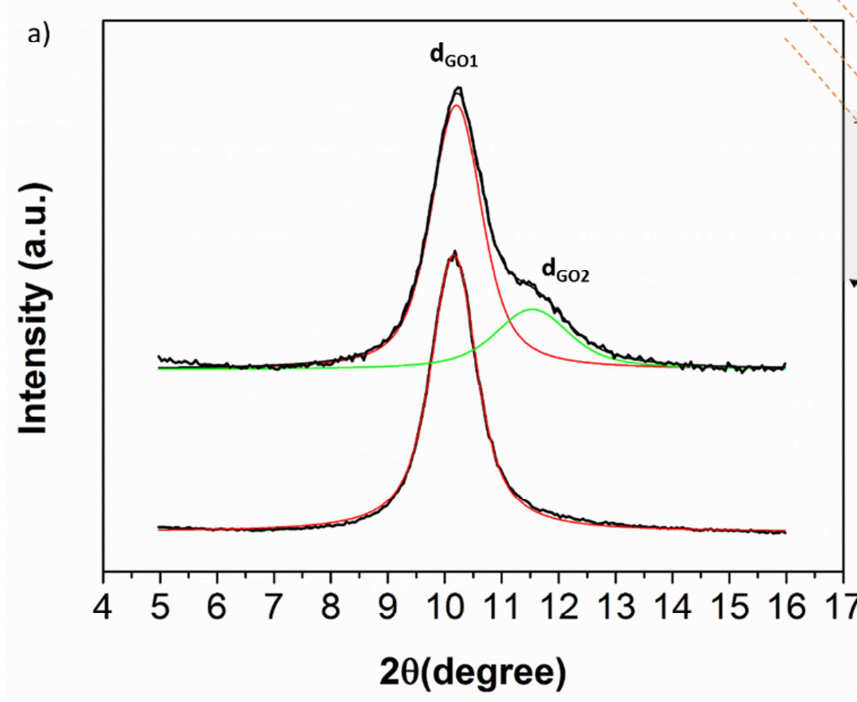

Interlayer water

Oxygen defects

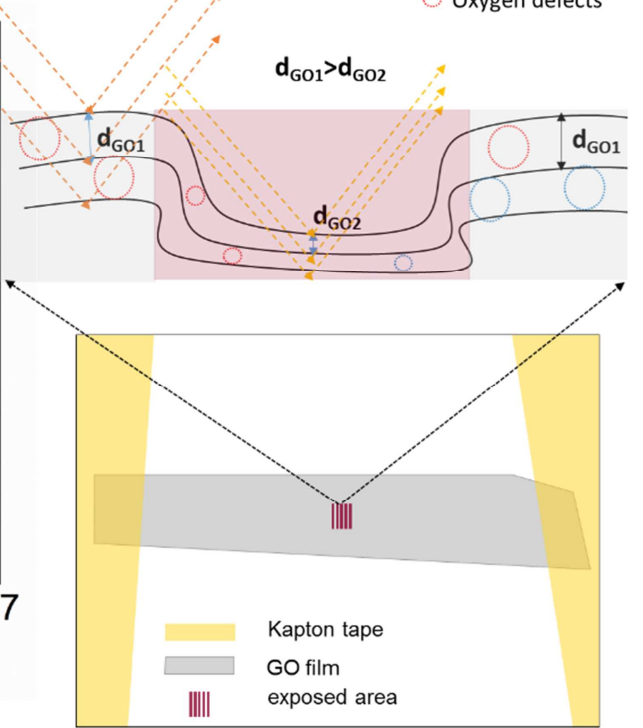

Figure 6. a) XRD of GO paper performed on the patterned and non-patterned regions by EBL. b) Schematic representation of the interlayer distance of GO sheets on the paper after electron beam exposure.

\subsection{Spectroscopic characterization of patterned GO paper}

XPS and FTIR spectra were recorded on the patterned $(500 \times 500 \mu \mathrm{m})$ and nonpatterned regions of the GO paper (Figure 7a)). The C1s XPS spectra were well fitted with 3 Lorentzian-Gaussian peaks which were assigned to $\mathrm{C} \operatorname{sp}^{2}(\sim 284.4 \mathrm{eV}), \mathrm{C}-\mathrm{O}$ $(286.6 \mathrm{eV})$ and $\mathrm{C}=\mathrm{O}(288.3 \mathrm{eV})[8]$ as showed on Figure $7 \mathrm{~b})$. In the patterned region, an increase of $20 \%$ in $\mathrm{sp}^{2}$ carbon was obtained corresponding to the restauration of the graphene carbon structure due to the elimination of oxygen functional groups (estimated loss of $16 \%$ for $\mathrm{C}-\mathrm{O}$ bonds and $4 \%$ for $\mathrm{C}=\mathrm{O}$ ) (Table 2 ). The $\mathrm{O} 1 \mathrm{~s}$ broad peaks at $\sim 532.3$ $\mathrm{eV}$, corresponding to the different oxygen functional groups $\mathrm{C}-\mathrm{O}$ and $\mathrm{C}=\mathrm{O}$ contributions [65] (Figure 7b) showed a clear decrease of intensity in the patterned region $(\sim 23 \%)$. The relative ratio between carbon and oxygen $(\mathrm{C} / \mathrm{O})$ in the patterned and non-patterned regions is 4.3 and 2.7 , respectively. The reduction of the GO films by EUV lithography using $200 \mathrm{~mJ} / \mathrm{cm}^{2}$ doses was reported showing a photoreduction 
efficiency of, $6 \%$ increase of $\mathrm{sp}^{2}$ carbon bonds and $20 \%$ decrease of $\mathrm{C}-\mathrm{O}$ bonds [41].

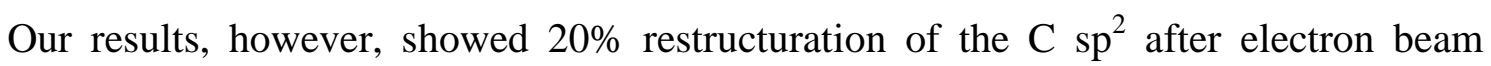
doses of $5 \times 10^{19}\left(\mathrm{e}^{-} / \mathrm{cm}^{2}\right)$. Recently, the same authors reported the GO photoreduction with VUV synchrotron radiation, at different radiation ranges, EUV (photon energy: 21 $\mathrm{eV}$ and $45 \mathrm{eV}$ ), and soft- $\mathrm{X}$ rays (photon energy: $628 \mathrm{eV}$ ), with a final $\mathrm{I}(\mathrm{C}-\mathrm{O}) / \mathrm{I}\left(\mathrm{C} \mathrm{sp}^{2}\right)$ ratio of $\sim 0.65[42]$, higher than the one obtained in our work $\left[\mathrm{I}(\mathrm{C}-\mathrm{O}) / \mathrm{I}\left(\mathrm{C} \mathrm{sp}^{2}\right)\right.$ ratio of 0.47]. It was also observed that EBL showed higher reduction efficiency of the GO films than the thermal treatment at $80{ }^{\circ} \mathrm{C}$ during 9 days (only small changes of $\mathrm{C} / \mathrm{O}$ ratio were observed)[66] and at $200{ }^{\circ} \mathrm{C}$ during 30 minutes $(\mathrm{C} / \mathrm{O}=3.9)$ [67]. The reduction mechanism of GO paper by EBL is not totally clear, however, based on the high energy of the electron beam used for the exposure, it is predictable that the major transformations occur by the decomposition of the oxygen functional groups into $\mathrm{H}_{2} \mathrm{O}$ and $\mathrm{O}_{2}$, with the simultaneous restructuration of the $\mathrm{C} \mathrm{sp}^{2}$ lattice. Theoretical calculations indicated that $\mathrm{C}-\mathrm{OH}$ and $\mathrm{C}-\mathrm{O}-\mathrm{C}$ groups can react between them to form $\mathrm{H}_{2} \mathrm{O}$ by overcoming the energy barrier of $0.5 \mathrm{eV}$ and C-O-C groups can react between them to form $\mathrm{O}_{2}$ at a barrier energy of $1.0 \mathrm{eV}$ [68]. However, it was also described that reactions with carbonyl groups occur through higher energy states $(2.33 \mathrm{eV}),[68]$ which is in accordance with the lower removal percentage of this functional group observed on our XPS data. 

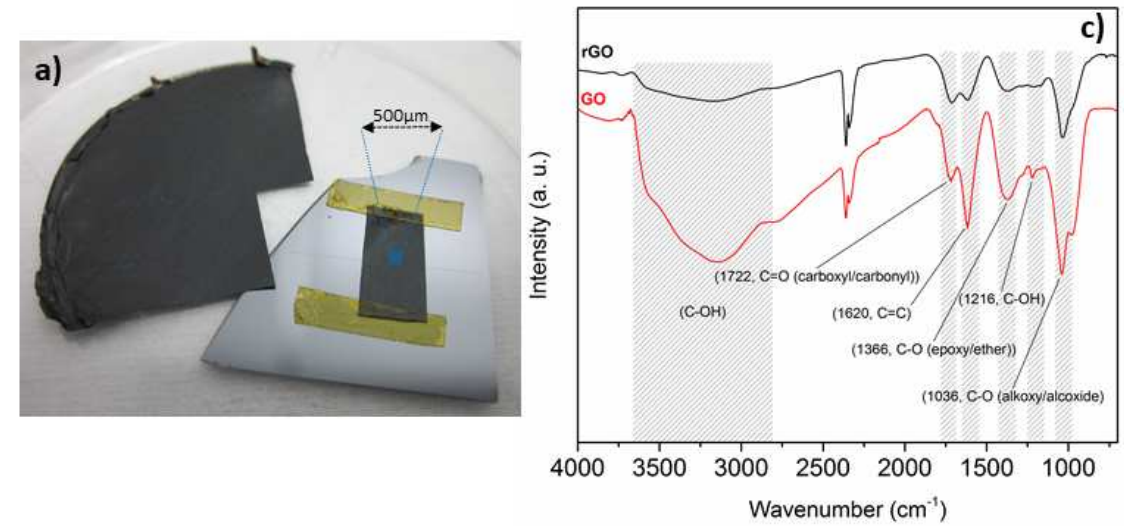

b)
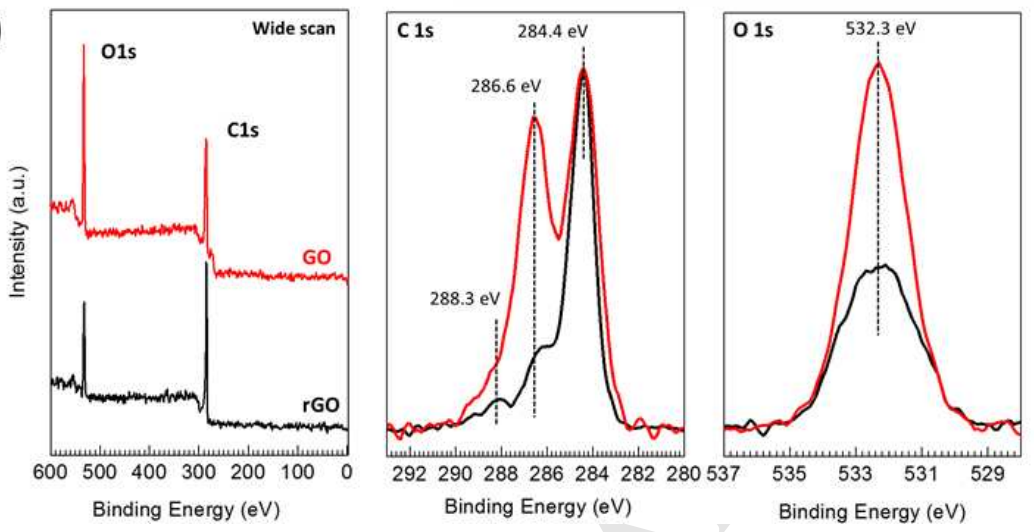

Figure 7. a) GO papers with patterned area of $500 \mu \mathrm{m}^{2}$ by EBL (blue region). b) XPS and c) FTIR spectra of the GO paper on the patterned region (rGO) and outside of the patterned region $(\mathrm{GO})$.

Table 2. Quantitative data obtained from the XPS spectra for the patterned and nonpatterned regions of GO paper.

\begin{tabular}{|c|c|c|c|c|c|c|}
\hline & & C1s fit & & & & \\
\hline Sample region & Component & $\mathrm{BE}(\mathrm{eV})$ & FWHM (eV) & Functional group & $\%$ & \\
\hline \multirow{3}{*}{ patterned } & 1 & 284.4 & 1.1 & $\mathrm{C} \mathrm{Sp}^{2}$ & 68 & \multirow{3}{*}{4.3} \\
\hline & 2 & 286.1 & 1.8 & $\mathrm{C}-\mathrm{O}$ & 24 & \\
\hline & 3 & 288.4 & 2.1 & $\mathrm{C}=\mathrm{O}$ & 8 & \\
\hline \multirow{3}{*}{ Non-patterned } & 1 & 284.4 & 1.5 & $\mathrm{C} \mathrm{Sp}^{2}$ & 48 & \multirow{3}{*}{2.7} \\
\hline & 2 & 286.5 & 1.6 & $\mathrm{C}-\mathrm{O}$ & 40 & \\
\hline & 3 & 288.0 & 2.5 & $\mathrm{C}=\mathrm{O}$ & 12 & \\
\hline
\end{tabular}


Regarding FTIR spectra, from a global overview of Figure 7c), it is clear that after electron beam exposure (red line, $\mathrm{rGO}$ ), there is a decrease in the intensity of the bands assigned to oxygen functional groups attached to the graphene basal plane. The intensity of the band assigned to the O-H stretch in hydroxyl groups $\left(\sim 3400 \mathrm{~cm}^{-1}\right)$ suffers a drastic decrease in $\mathrm{rGO}$ due to the release of water intercalated between the GO planes and defective regions and hydroxyl desorption; hydroxyl groups may also be released from the basal plane by the formation of water molecules [21]. Carboxyl groups stretch band at $1620 \mathrm{~cm}^{-1}$ (that also has a contribution from the bending vibration of intercalated water) shows also a very strong decrease in intensity in rGO (Figure 7c) red line), that is more evident when compared with the carbonyl stretch band at $1722 \mathrm{~cm}^{-1}$ (that was of much lower intensity in GO, Figure 7c) black line). The FTIR results clearly confirm the preferential elimination of hydroxyl and carboxyl groups from the GO basal plane on the reduction process, as previously reported [21]. For carbonyl groups stretch band at $1722 \mathrm{~cm}^{-1}$ no significant intensity changes were observed, which demonstrates the higher stability of these groups in the GO structure. These observations are consistent with the ones obtained from XPS data.

Raman spectroscopy is a very sensitive approach for the structural characterization of GO and its reduced derivatives. Figure 8a) shows the Raman spectral image of the patterned GO paper with different contrast regions, the light coloured lines corresponding to the reduced patterns with $10 \mu \mathrm{m}$ periodicity. Raman images were obtained by accumulating a full Raman spectrum at each pixel, and constructed by integrating over a specific Raman band. Figure 8b) shows the Raman spectra corresponding to the reduced pattern lines (medium spectrum in the violet area) and the non-reduced broader lines (medium spectrum in the green area) where it is possible to observe the $\mathrm{D}$ band at $1350 \mathrm{~cm}^{-1}$, which is known to be characteristic of structural 
disorder (induced by oxidation), and $\mathrm{G}$ band at $1585 \mathrm{~cm}^{-1}$, that is associated to the structural graphitic order, and the broad band 2D with maxima centred around 2700 and $2900 \mathrm{~cm}^{-1}$ [69]. The colour contrast between the reduced and non-reduced lines visible on Figure 8a), was obtained by considering the Raman integrated intensity at the dashed region in Figure 8 b), corresponding to the D band shoulder at $\sim 1200 \mathrm{~cm}^{-1}$ correlated with the carbon structure of GO materials. Recently, Claramunt et al. [69] revealed the importance of the interbands on the interpretation of GO Raman spectrum. The authors fitted GO Raman spectrum using five functions that can be attributed to $G, D^{\prime}, D^{\prime}, D^{*}$ and $\mathrm{D}^{\prime \prime}$ bands, however in our case the best fitting was achieved with four Voigt functions that correspond to G, D, D* and $\mathrm{D}^{\prime \prime}$ bands, as shown in Figure 8c) and d). These authors observed that the decrease of oxygen content is correlated with the blue shift of the $\mathrm{D}^{*}$ maximum. Our results show that the patterned lines have a $\mathrm{D}^{*}{ }_{\max }$ at 1258 $\mathrm{cm}^{-1}$ and the non-patterned region a $\mathrm{D}^{*}{ }_{\max }$ at $1238 \mathrm{~cm}^{-1}$ that corresponds exactly to a decrease of oxygen content due to the electron beam exposure. The $\mathrm{I}_{\mathrm{D}} / \mathrm{I}_{\mathrm{G}}$ ratios observed (the average ratio across the purple and green lines on the mapping) presented a slight variation between the two different regions $[69,70]$. 

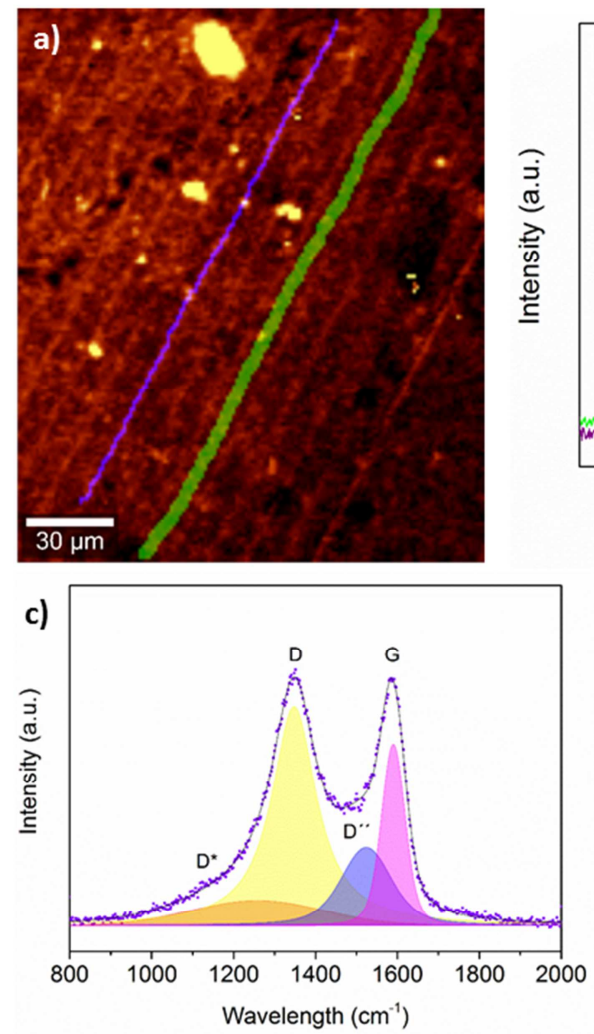
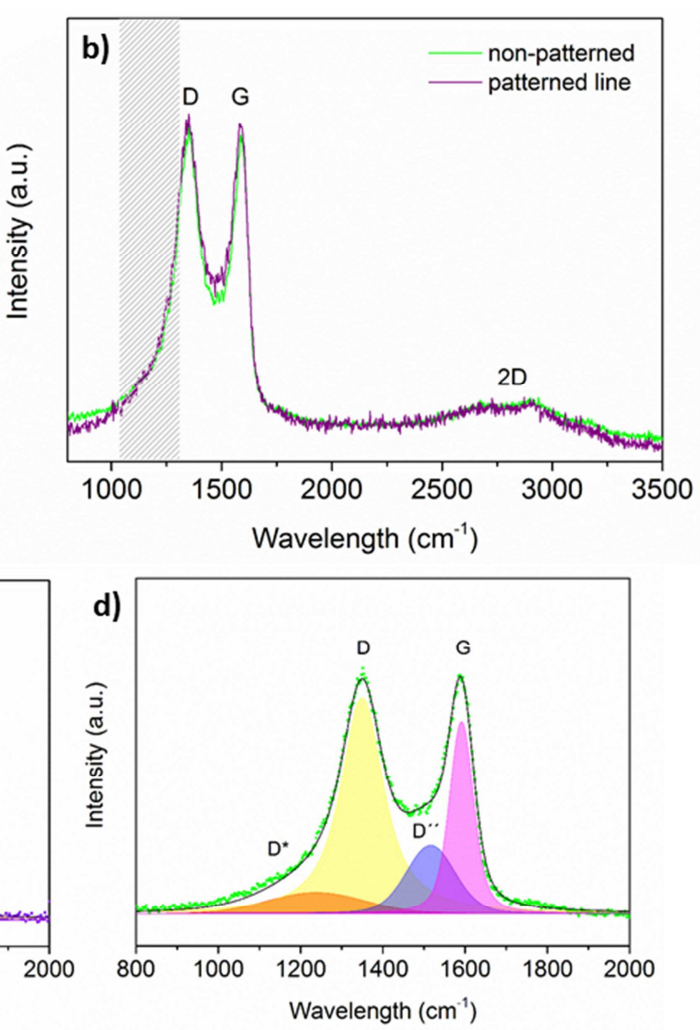

Figure 8. a) Raman image of the patterned region of the GO paper, obtained using the integrated intensity of the dashed region in $\mathrm{b}\left(\sim 1100-1300 \mathrm{~cm}^{-1}\right)$; areas with reduced (patterned) and non-reduced GO (non-patterned) were marked by the violet and the green lines, respectively. b) Raman spectra obtained by the medium spectra of all pixels in the respective selected areas in (a), violet for patterned GO and green for nonpatterned GO. Fitting of the D and G peaks is shown in c) for patterned GO and in d) for non-patterned GO.

\subsection{Electrical properties of patterned GO paper}

The electrical properties of the material were characterized first by conductive AFM. A local resistivity of $1000 \Omega \cdot \mathrm{m}$ was measured. While the resistivity within the limits of the exposed area was much lower than reported values for raw GO, [71] no significant difference in resistivity was observed between the exposed lines and the space between the lines (Figure S2 - supplementary information). This apparent contradiction with the 
results of previous characterization techniques could be explained by a secondary heating of the area immediately around that exposed to the electron beam, which would anneal that region, therefore reducing the contrast between exposed and unexposed areas.

The electrical conductivity was also measured, before and after exposure, between patterned metal contacts. First, a line of $2 \mu \mathrm{m}$ and $800 \mu \mathrm{m}$ of length is patterned by electron beam exposure with dose $10000 \times 10^{3} \mu \mathrm{C} / \mathrm{cm}^{2}$. A second electron exposure was performed using a $500 \mathrm{~nm}$ thick layer of PMMA to draw contacts separated by 100 $\mu \mathrm{m}$ (Figure S3 - supplementary information). The electron dose used to expose PMMA is $800 \mu \mathrm{C} / \mathrm{cm}^{2}$, which, according to the results in table 1 (sample 1), has no effect on GO reduction. After development using methyl-isobutyl ketone (MIBK), a layer of Cr 3 $\mathrm{nm} / \mathrm{Au} 20 \mathrm{~nm}$ was deposited. Acetone was used to perform the lift-off. The resistance of the line was measured by applying $1 \mu \mathrm{A}$ between the gold contacts, and a value of $140 \mathrm{k} \Omega$ was obtained. The resistance of the measurement circuit, including contact resistance, was $80 \Omega$. Assuming that the GO becomes homogeneously conductive inside the volume exposed to the electron beam, that the surrounding GO is insulating, and using a thickness of $70 \mu \mathrm{m}$ for the exposed rGO paper, the resistivity of the exposed material is estimated to be $0.44 \Omega \cdot \mathrm{m}$. During the same fabrication process, two rectangular contacts were prepared on an area not exposed to the electron beam reduction process. The rectangles measured $600 \mu \mathrm{m}$ in width and were separated by 140 $\mu \mathrm{m}$. Using a pico-ampmeter and applying $50 \mathrm{~V}$, the resistance of the GO paper between the two rectangular contacts was measured to be $81 \mathrm{M} \Omega$. Neglecting the current not flowing straight between the two contacts, and using a paper thickness of $40 \mu \mathrm{m}$, the resistivity of the unexposed GO is $14 \mathrm{k} \Omega \cdot \mathrm{m}$. From these measurements in the exposed 
and unexposed areas, the exposure to the electron reduction process increases the conductivity by a factor of $3 \times 10^{4}$.

\subsection{Mechanical analysis of patterned GO paper}

In order to further understand the structural effects of electron beam exposition on the reduced patterned surface of GO paper, nanoindentation tests were performed (Figure 9). Two different positions were tested, on the unmodified surface and on the square patterned surface by electron beam at $100 \mathrm{keV}$ (Figure 9a)). The nanoindentation corresponds to the load-displacement curves, each containing 16 loading-unloading cycles (Figure 9b)). From the nanoindentation experiments it is possible to obtain hardness $(\mathrm{H})$ and the Elastic modulus (E) at different penetration depth [72].

a)
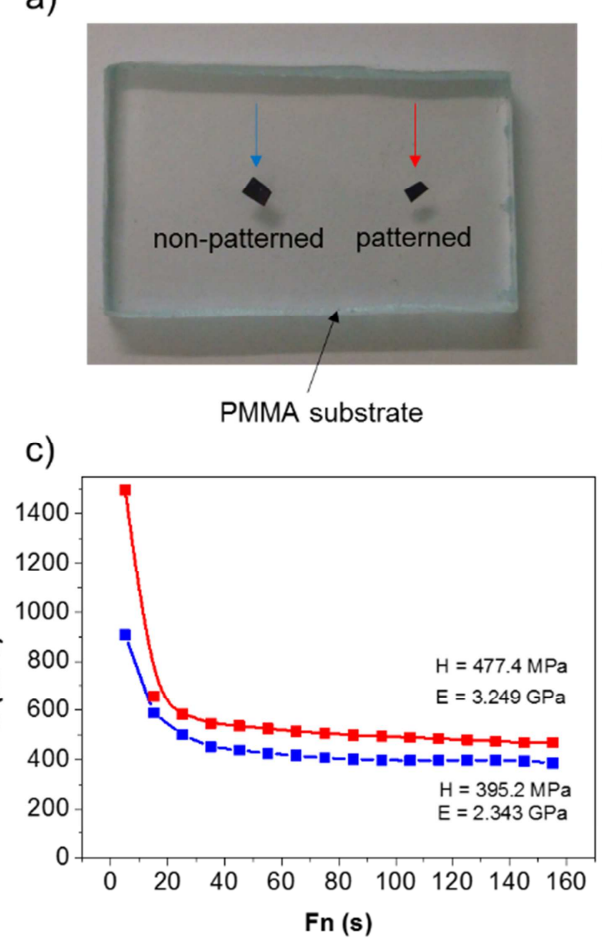

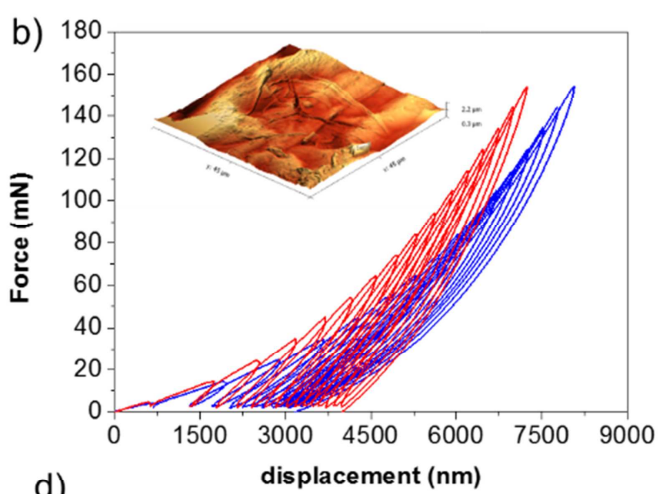

d)

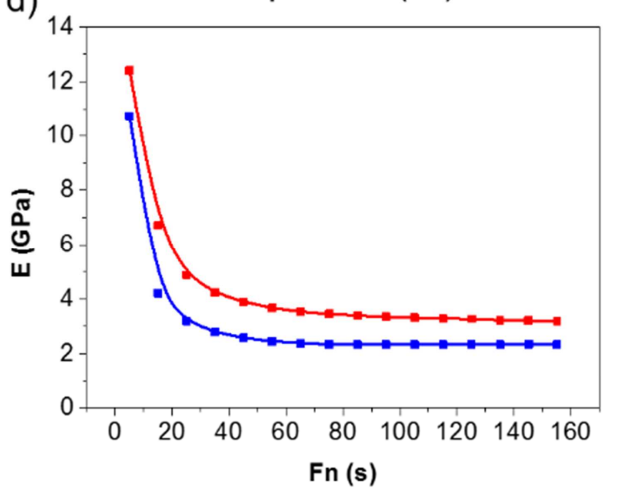

Figure 9. Representative load-displacement curves obtained for the GO papers on the patterned and non-patterned regions by EBL. 
A very interesting feature of the data obtained shows that the $\mathrm{H}$ and $\mathrm{E}$ decrease with the increase of applied forces (for values $<35 \mathrm{mN}$ ). Those results can be attributed to the nanoscale bending effects of the GO paper or/and to the decrease of the GO interlayers distance until reaching the maximum equilibrium limit to the rupture, which is characteristic to their layer-by-layer hierarchical structure, combining intralayer strong $\mathrm{sp}^{2}$ bonds and interlayer crosslinks for efficient load transfer [73]. These effects could be reduced when the tests were performed using a delay time between indentations $(10$, 100 and 500 s), however it promotes other constraints (Figure S4 - supplementary information). After that value of force, it was observed that $\mathrm{H}$ and $\mathrm{E}$ are independent of the penetration depth on the modified $(\mathrm{H}=477.4 \mathrm{MPa}$ and $\mathrm{E}=3.249 \mathrm{GPa})$ and unmodified $(\mathrm{H}=395.2 \mathrm{MPa}$ and $\mathrm{E}=2.343 \mathrm{GPa}) \mathrm{GO}$ paper surface. In fact, the results showed higher values of $\mathrm{H}$ and $\mathrm{E}$ for the reduced patterned regions, which means that the electron beam doesn't destroy the carbon structure and also suggest that an effective reduction of GO sheets occurs allowing the decrease of the interlayer distance, thus producing a more compact material. It could be predicted that the exposure at $100 \mathrm{keV}$ might start damaging the carbon network since it is above the threshold (93 keV) for C$\mathrm{C}$ breaking, however it is not probable that as many electrons as those that reach the surface lose enough energy on one single collision to significantly damage the network. It was already observed that the thermal reduction of the GO paper promotes an important increase of $\mathrm{E}$ and $\mathrm{H}$ [74].

\subsection{Cytocompatibility of GO patterned papers}

Preliminary in vitro biocompatibility tests of the GO paper were carried out with murine L929 fibroblasts, an immortalized mammalian cell line commonly used for biocompatibility assessment of materials in vitro [75]. Cells were seeded on both patterned and non-patterned areas of the samples and cultures were evaluated at sub- 
confluence $(96 \mathrm{~h})$ to facilitate the investigation of adhesion and morphology parameters. Figure 10 illustrates representative micrographs of L929 cells grown on GO paper by both CLSM and SEM. As it can be appreciated, fibroblasts grew on the substrate independently of the presence of the pattern, forming cultures of homogeneous cell distribution and abundant cells undergoing mitosis that were indicative of active cell proliferation (Figure 10b). Importantly, cells growing on the polystyrene well at the periphery of the GO papers also displayed healthy morphological features (spread phenotype and active proliferation) even in the presence of small portions of GO released from the film borders (Figure $10 \mathrm{c}$ )). It is worth noting that, despite the potent antibacterial properties described for GO [76], these substrates did not induce significant morphological alterations on L929 fibroblasts, a study model of mammalian eukaryotic cells. To further investigate these cellular processes, SEM studies were performed. Fibroblasts homogeneously colonized the substrate preserving their typical morphology and displaying a more or less spread aspect depending on the phase of the cell cycle (Figure 10d)). As previously confirmed by CLSM, cells in active proliferation were evident, with a common rounded shape to enable detachment from the substrate and division (Figure 10e)). Abundant cytoplasmic projections such as filopodia (thin and long) and lamellipodia (wide and large) were observed in close contact with the GO paper surface (Figure 10f)). These cell extensions are typical of migrating cells, involve the participation of the actin-based cytoskeleton and play a crucial role in adhesion processes.

Taken together, these results evidence that the patterned GO paper fabricated is cytocompatible with mammalian cells, supporting cell adhesion, growth and proliferation and preserving cell morphology. Contrary to findings with bacteria [76], the presence of GO in these samples does not seem to have a negative impact on their 


\section{ACCEPTED MANUSCRIPT}

cytocompatibility with mammalian eukaryotic cells. Moreover, EBL seems to be an useful technique for the fabrication of patterned substrates for biomedical applications, as also demonstrated for other patterning strategies such as laser interference.[77] The cytocompatible nature of these samples encourages further investigation with these materials, as nanotopographical substrates have revealed ability to induce and modulate specific cell responses such as stem cell self-renewal and multipotency [78].
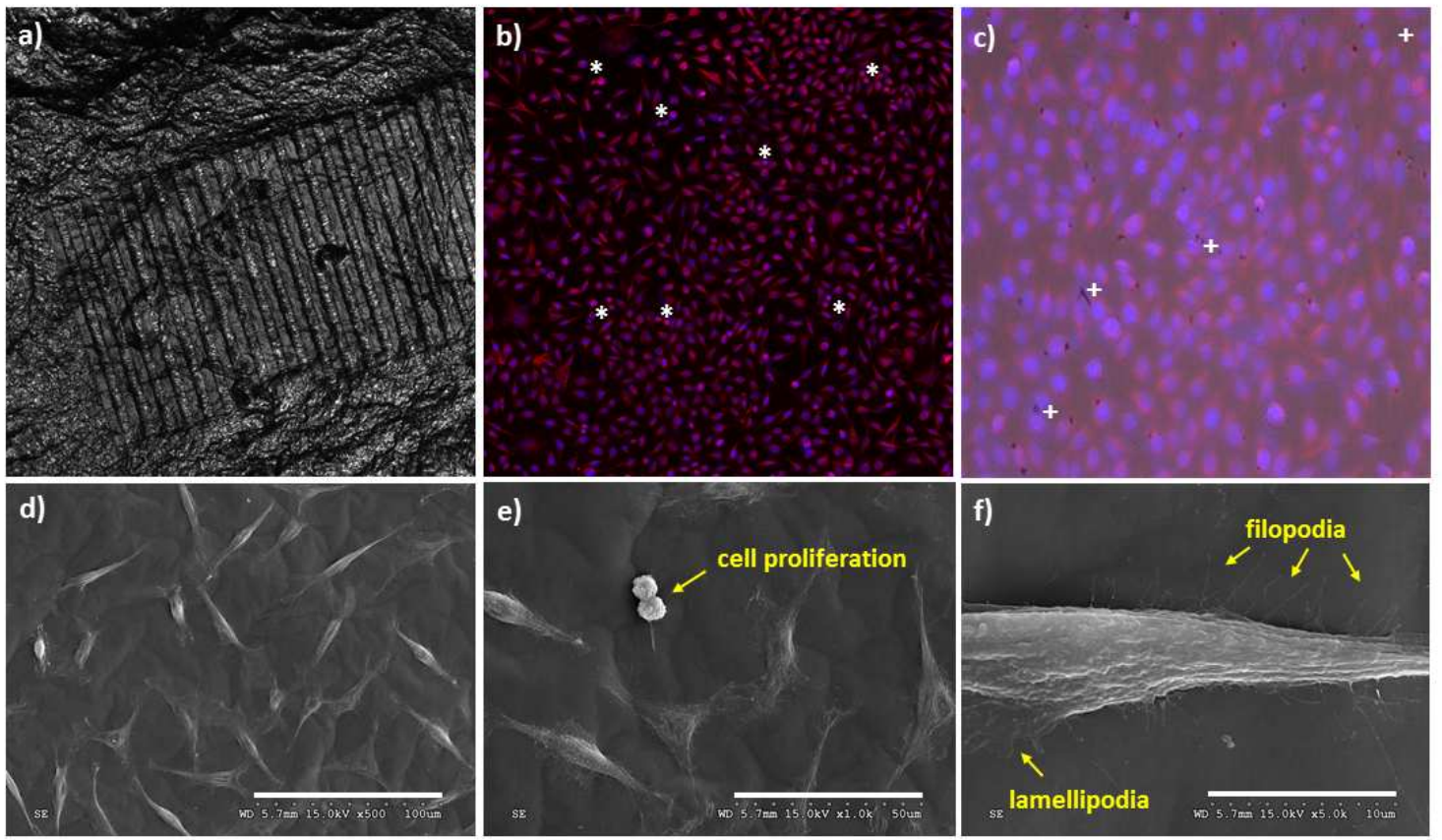

Figure 10. Cytocompatibility in vitro studies of L929 fibroblasts cultured on GO paper. CLSM micrograph in reflexion mode illustrating the pattern position in the film a). Adhesion and cytoskeletal visualization by CLSM on both patterned and non-patterned areas b) and at the periphery of the GO paper on polystyrene (c). Cells in active proliferation are labelled with white asterisks and GO film fragments with white crosses. Scale bars: $150 \mu \mathrm{m}(\mathrm{a}, \mathrm{b})$ and $50 \mu \mathrm{m}$ c). Morphological studies by SEM (d-f). Cells undergoing cytokinesis and detailed cytoplasmic projections are indicated by yellow arrows. Scale bars: 100, 50 and $10 \mu \mathrm{m}(\mathrm{d}$, e and f, respectively). 


\section{Conclusions}

We explored the patterning design of GO paper substrates using direct-write EBL to create nanoscale conductive lines, using a $100 \mathrm{keV}$ electron beam. This new approach revealed to be very effective and versatile for the design of reduced patterns on the GO papers surface, allowing for free pattern choice and use of different electron doses and beam step sizes. The contrast of the reduced stripes in the patterned regions increased with the increase of electron beam doses. Structural analysis of the patterned paper showed that the interlayer distance between GO sheets decreases after reduction, allowing to increase the Hardness and Young modulus, which enables the manipulation and integration of this material on different devices. Furthermore, we also observed that exposed areas to electron beam reduction process showed an increase in the electrical conductivity of up to $3 \times 10^{4}$ times. Table 3 summarize the main structural properties observed for the patterned and non-patterned regions of GO paper.

Table 3. Resume of the structural properties of reduced patterned GO paper by ELB in comparison to non-modified GO paper

\begin{tabular}{|l|c|c|c|c|c|}
\hline \multirow{2}{*}{ GO patterned paper } & \multicolumn{5}{|c|}{ Structural characterization } \\
\cline { 2 - 6 } & XRD & Raman & XPS & Resistivity & Nanoindentation \\
\hline $\begin{array}{l}\text { non-patterned } \\
\text { region }\end{array}$ & $2 \theta=10.15^{\circ}(\mathrm{d} \sim .7 \AA \AA)$ & $\mathrm{D}^{*}{ }_{\max }=1238 \mathrm{~cm}^{-1}$ & $\mathrm{C} 1 \mathrm{~s} / \mathrm{O} 1 \mathrm{~s}$ ratio $=4.3$ & $14 \mathrm{~K} \Omega \mathrm{m}$ & $\begin{array}{c}\mathrm{H}=395.2 \mathrm{MPa} \\
\mathrm{E}=2.343 \mathrm{GPa}\end{array}$ \\
\hline patterned region* & $\begin{array}{l}2 \theta=10.20^{\circ}(\mathrm{d} \sim 8.7 \AA) \\
2 \theta=11.53^{\circ}(\mathrm{d} 8.7 \AA \AA)\end{array}$ & $\mathrm{D}^{*}{ }_{\max }=1258 \mathrm{~cm}^{-1}$ & $\mathrm{C} 1 \mathrm{~s} / 01 \mathrm{~s}$ ratio=2.7 & $0.44 \Omega \mathrm{m}$ & $\begin{array}{l}\mathrm{H}=477.4 \mathrm{MPa} \\
\mathrm{E}=3.249 \mathrm{GPa}\end{array}$ \\
\hline
\end{tabular}

*exposure ELB experimental conditions number 5 reported on table 1

Cytocompatibility tests in vitro with L929 fibroblasts evidenced the ability of the patterned GO paper fabricated to support mammalian cell adhesion, growth and proliferation. Abundant cytoplasmic projections, such as filopodia and lamellipodia, were observed in close contact with the GO paper surface. 


\section{Ackowledgements}

Gil Gonçalves thanks the Fundação para a Ciência e Tecnologia (FCT) for the PostDoc grant (SFRH/BDP/84419/2012).

P.A.A.P.M. acknowledge the FCT/MCTES for a research contract under the Program Investigador 2013 (IF/00917/2013/CP1162/CT0016) and TEMA - Centre for Mechanical Technology and Automation (UID/EMS/00481/2013), financed by national funds through the FCT/MEC. I.B. wish to acknowledge the Portuguese Foundation for Science and Technology for the financial support (grant IF/00582/2015).

H.I.S.N. acknowledges CICECO-Aveiro Institute of Materials, POCI-01-0145-FEDER007679 (FCT Ref. UID /CTM /50011/2013), financed by national funds through the FCT/MEC and when appropriate co-financed by FEDER under the PT2020 Partnership Agreement.

The biological studies of this work have been funded by the Ministerio de Economía y Competitividad and the Fondo Europeo de Desarrollo Regional (MAT2016-78857-R, MINECO/FEDER, UE). AGM and MCS acknowledge ISCIII-MINECO-FEDER for respective contracts. Authors would like to thank Dr M. Teresa Portolés from the Biochemistry and Molecular Biology Department at Universidad Complutense de Madrid for the generous supply of L929 fibroblasts. Dr José Ángel Rodríguez and Dr Javier Mazarío from the Service of Microscopy and Image Analysis at the Hospital Nacional de Parapléjicos are acknowledged for assistance with CLSM studies and Dr Enrique Rodríguez from the Servicio Interdepartamental de Investigación at the Universidad Autónoma de Madrid for SEM studies. 


\section{References}

[1] S. Park, R.S. Ruoff, Chemical methods for the production of graphenes, Nat. Nanotechnol. 4(4) (2009) 217-224.

[2] D.C. Marcano, D.V. Kosynkin, J.M. Berlin, A. Sinitskii, Z. Sun, A. Slesarev, L.B. Alemany, W. Lu, J.M. Tour, Improved Synthesis of Graphene Oxide, ACS Nano 4(8) (2010) 4806-4814.

[3] D.R. Dreyer, S. Park, C.W. Bielawski, R.S. Ruoff, The chemistry of graphene oxide, Chem. Soc. Rev. 39(1) (2010) 228-240.

[4] J.I. Paredes, S. Villar-Rodil, A. Martinez-Alonso, J.M.D. Tascon, Graphene oxide dispersions in organic solvents, Langmuir 24(19) (2008) 10560-10564.

[5] H.Y. He, J. Klinowski, M. Forster, A. Lerf, A new structural model for graphite oxide, Chem. Phys. Lett. 287(1-2) (1998) 53-56.

[6] A.M. Dimiev, L.B. Alemany, J.M. Tour, Graphene Oxide. Origin of Acidity, Its Instability in Water, and a New Dynamic Structural Model, ACS Nano 7(1) (2013) $576-588$.

[7] S. Kim, S. Zhou, Y. Hu, M. Acik, Y.J. Chabal, C. Berger, W. de Heer, A. Bongiorno, E. Riedo, Room-temperature metastability of multilayer graphene oxide films, Nat. Mater. 11(6) (2012) 544-549.

[8] S. Stankovich, D.A. Dikin, R.D. Piner, K.A. Kohlhaas, A. Kleinhammes, Y. Jia, Y. Wu, S.T. Nguyen, R.S. Ruoff, Synthesis of graphene-based nanosheets via chemical reduction of exfoliated graphite oxide, Carbon 45(7) (2007) 1558-1565.

[9] C. Gomez-Navarro, J.C. Meyer, R.S. Sundaram, A. Chuvilin, S. Kurasch, M. Burghard, K. Kern, U. Kaiser, Atomic Structure of Reduced Graphene Oxide, Nano Lett. 10(4) (2010) 1144-1148. 
[10] S. Pei, H.-M. Cheng, The reduction of graphene oxide, Carbon 50(9) (2012) 32103228.

[11] S. Nardecchia, D. Carriazo, M. Luisa Ferrer, M.C. Gutierrez, F. del Monte, Three dimensional macroporous architectures and aerogels built of carbon nanotubes and/or graphene: synthesis and applications, Chem. Soc. Rev. 42(2) (2013) 794830.

[12] H.-P. Cong, J.-F. Chen, S.-H. Yu, Graphene-based macroscopic assemblies and architectures: an emerging material system, Chem. Soc. Rev. 43(21) (2014) 72957325.

[13] D.A. Dikin, S. Stankovich, E.J. Zimney, R.D. Piner, G.H.B. Dommett, G. Evmenenko, S.T. Nguyen, R.S. Ruoff, Preparation and characterization of graphene oxide paper, Nature 448(7152) (2007) 457-460.

[14] Y. Xu, K. Sheng, C. Li, G. Shi, Self-Assembled Graphene Hydrogel via a OneStep Hydrothermal Process, ACS Nano 4(7) (2010) 4324-4330.

[15] C. Cheng, D. Li, Solvated Graphenes: An Emerging Class of Functional Soft Materials, Adv. Mater. 25(1) (2013) 13-30.

[16] L. Qiu, X.H. Zhang, W.R. Yang, Y.F. Wang, G.P. Simon, D. Li, Controllable corrugation of chemically converted graphene sheets in water and potential application for nanofiltration, Chem. Comm. 47(20) (2011) 5810-5812.

[17] X.W. Yang, L. Qiu, C. Cheng, Y.Z. Wu, Z.F. Ma, D. Li, Ordered Gelation of Chemically Converted Graphene for Next-Generation Electroconductive Hydrogel Films, Angew. Chem. Int. Ed. 50(32) (2011) 7325-7328. 
[18] X.W. Yang, J.W. Zhu, L. Qiu, D. Li, Bioinspired Effective Prevention of Restacking in Multilayered Graphene Films: Towards the Next Generation of HighPerformance Supercapacitors, Adv. Mater. 23(25) (2011) 2833-+.

[19] G.K. Wang, X. Sun, F.Y. Lu, H.T. Sun, M.P. Yu, W.L. Jiang, C.S. Liu, J. Lian, Flexible Pillared Graphene-Paper Electrodes for High-Performance Electrochemical Supercapacitors, Small 8(3) (2012) 452-459.

[20] D.T. Pham, T.H. Lee, D.H. Luong, F. Yao, A. Ghosh, V.T. Le, T.H. Kim, B. Li, J. Chang, Y.H. Lee, Carbon Nanotube-Bridged Graphene 3D Building Blocks for Ultrafast Compact Supercapacitors, ACS Nano 9(2) (2015) 2018-27.

[21] P.V. Kumar, N.M. Bardhan, S. Tongay, J. Wu, A.M. Belcher, J.C. Grossman, Scalable enhancement of graphene oxide properties by thermally driven phase transformation, Nat. Chem. 6(2) (2014) 151-158.

[22] B. Florian, Irradiation effects in carbon nanostructures, Rep. Prog. in Phys. 62(8) (1999) 1181.

[23] A.V. Krasheninnikov, F. Banhart, Engineering of nanostructured carbon materials with electron or ion beams, Nat. Mater. 6(10) (2007) 723-733.

[24] A.V. Krasheninnikov, K. Nordlund, Ion and electron irradiation-induced effects in nanostructured materials, J. of Appl. Phys. 107(7) (2010).

[25] K.S. Subrahmanyam, P. Kumar, A. Nag, C.N.R. Rao, Blue light emitting graphenebased materials and their use in generating white light, Solid State Commun. 150(37-38) (2010) 1774-1777.

[26] Y. Matsumoto, M. Koinuma, S.Y. Kim, Y. Watanabe, T. Taniguchi, K. Hatakeyama, H. Tateishi, S. Ida, Simple Photoreduction of Graphene Oxide 
Nanosheet under Mild Conditions, ACS Appl. Mater. Interfaces 2(12) (2010) 34613466.

[27] V.A. Smirnov, A.A. Arbuzov, Y.M. Shul'ga, S.A. Baskakov, V.M. Martynenko, V.E. Muradyan, E.I. Kresova, Photoreduction of graphite oxide, High Energ. Chem. 45(1) (2011) 57-61.

[28] Y. Zhou, Q. Bao, B. Varghese, L.A.L. Tang, C.K. Tan, C.-H. Sow, K.P. Loh, Microstructuring of Graphene Oxide Nanosheets Using Direct Laser Writing, Adv. Mater. 22(1) (2010) 67-+.

[29] L.J. Cote, R. Cruz-Silva, J. Huang, Flash Reduction and Patterning of Graphite Oxide and Its Polymer Composite, Journal of the American Chemical Society 131(31) (2009) 11027-11032.

[30] R. Kumar, R. Savu, E. Joanni, A.R. Vaz, M.A. Canesqui, R.K. Singh, R.A. Timm, L.T. Kubota, S.A. Moshkalev, Fabrication of interdigitated micro-supercapacitor devices by direct laser writing onto ultra-thin, flexible and free-standing graphite oxide films, RSC Advances 6(88) (2016) 84769-84776.

[31] S. Gilje, S. Dubin, A. Badakhshan, J. Farrar, S.A. Danczyk, R.B. Kaner, Photothermal Deoxygenation of Graphene Oxide for Patterning and Distributed Ignition Applications, Advanced Materials 22(3) (2010) 419-423.

[32] P. Kumar, K.S. Subrahmanyam, C.N.R. Rao, Graphene Patterning and Lithography Employing Laser/Electron-Beam Reduced Graphene Oxide and Hydrogenated Graphene, Materials Express 1(3) (2011) 252-256.

[33] S. Prezioso, M. Perrozzi, M. Donarelli, F. Bisti, S. Santucci, L. Palladino, M. Nardone, E. Treossi, V. Palermo, L. Ottaviano, Large Area Extreme-UV 
Lithography of Graphene Oxide via Spatially Resolved Photoreduction, Langmuir 28(12) (2012) 5489-5495.

[34] L. Guo, H.-B. Jiang, R.-Q. Shao, Y.-L. Zhang, S.-Y. Xie, J.-N. Wang, X.-B. Li, F. Jiang, Q.-D. Chen, T. Zhang, H.-B. Sun, Two-beam-laser interference mediated reduction, patterning and nanostructuring of graphene oxide for the production of a flexible humidity sensing device, Carbon 50(4) (2012) 1667-1673.

[35] J.-N. Wang, R.-Q. Shao, Y.-L. Zhang, L. Guo, H.-B. Jiang, D.-X. Lu, H.-B. Sun, Biomimetic Graphene Surfaces with Superhydrophobicity and Iridescence, Chemistry - An Asian Journal 7(2) (2012) 301-304.

[36] S. Papazoglou, V. Tsouti, S. Chatzandroulis, I. Zergioti, Direct laser printing of graphene oxide for resistive chemosensors, Optics \& Laser Technology 82(Supplement C) (2016) 163-169.

[37] Y. 37, M. Koinuma, S.Y. Kim, Y. Watanabe, T. Taniguchi, K. Hatakeyama, H. Tateishi, S. Ida, Simple Photoreduction of Graphene Oxide Nanosheet under Mild Conditions, Acs Applied Materials \& Interfaces 2(12) (2010) 3461-3466.

[38] C. Petridis, Y.H. Lin, K. Savva, G. Eda, E. Kymakis, T.D. Anthopoulos, E. Stratakis, Post-fabrication, in situ laser reduction of graphene oxide devices, Applied Physics Letters 102(9) (2013).

[39] V.A. Smirnov, A.A. Arbuzov, Y.M. Shul'ga, S.A. Baskakov, V.M. Martynenko, V.E. Muradyan, E.I. Kresova, Photoreduction of graphite oxide, High Energy Chemistry 45(1) (2011) 57-61.

[40] S.Y. Zhou, C.O. Girit, A. Scholl, C.J. Jozwiak, D.A. Siegel, P. Yu, J.T. Robinson, F. Wang, A. Zettl, A. Lanzara, Instability of two-dimensional graphene: Breaking sp(2) bonds with soft x rays, Phys. Rev. B 80(12) (2009). 
[41] S. Prezioso, F. Perrozzi, M. Donarelli, F. Bisti, S. Santucci, L. Palladino, M. Nardone, E. Treossi, V. Palermo, L. Ottaviano, Large Area Extreme-UV Lithography of Graphene Oxide via Spatially Resolved Photoreduction, Langmuir 28(12) (2012) 5489-5495.

[42] S. Prezioso, F. Perrozzi, M. Donarelli, E. Stagnini, E. Treossi, V. Palermo, S. Santucci, M. Nardone, P. Moras, L. Ottaviano, Dose and wavelength dependent study of graphene oxide photoreduction with VUV Synchrotron radiation, Carbon 79 (2014) 478-485.

[43] M. Baraket, S.G. Walton, Z. Wei, E.H. Lock, J.T. Robinson, P. Sheehan, Reduction of graphene oxide by electron beam generated plasmas produced in methane/argon mixtures, Carbon 48(12) (2010) 3382-3390.

[44] S. Kim, D.D. Kulkarni, M. Henry, P. Zackowski, S.S. Jang, V.V. Tsukruk, A.G. Fedorov, Localized conductive patterning via focused electron beam reduction of graphene oxide, Appl. Phys. Lett. 106(13) (2015).

[45] E. Jin, J. He, K. Sheng, Z. Zhang, G. Shi, Q. Zheng, Electron-irradiation-induced reinforcement of reduced graphene oxide papers, Acta Mater. 61(17) (2013) 64666473.

[46] U.Y. Lau, S.S. Saxer, J. Lee, E. Bat, H.D. Maynard, Direct Write Protein Patterns for Multiplexed Cytokine Detection from Live Cells Using Electron Beam Lithography, ACS Nano 10(1) (2016) 723-729.

[47] P.-Y. Wang, W.-T. Li, J. Yu, W.-B. Tsai, Modulation of osteogenic, adipogenic and myogenic differentiation of mesenchymal stem cells by submicron grooved topography, J. Mater. Sci. Mater. Med 23(12) (2012) 3015-3028. 
[48] G. Shayan, N. Felix, Y. Cho, M. Chatzichristidi, M.L. Shuler, C.K. Ober, K.H. Lee, Synthesis and Characterization of High-Throughput Nanofabricated Poly(4Hydroxy Styrene) Membranes for In Vitro Models of Barrier Tissue, Tissue Eng. Part C: Methods 18(9) (2012) 667-676.

[49] A. Solanki, S.-T.D. Chueng, P.T. Yin, R. Kappera, M. Chhowalla, K.-B. Lee, Axonal Alignment and Enhanced Neuronal Differentiation of Neural Stem Cells on Graphene-Nanoparticle Hybrid Structures, Adv. Mater.25(38) (2013) 5477-5482.

[50] L.H. Hess, M. Jansen, V. Maybeck, M.V. Hauf, M. Seifert, M. Stutzmann, I.D. Sharp, A. Offenhäusser, J.A. Garrido, Graphene Transistor Arrays for Recording Action Potentials from Electrogenic Cells, Adv. Mater. 23(43) (2011) 5045-5049.

[51] T.R. Nayak, H. Andersen, V.S. Makam, C. Khaw, S. Bae, X. Xu, P.-L.R. Ee, J.-H. Ahn, B.H. Hong, G. Pastorin, B. Özyilmaz, Graphene for Controlled and Accelerated Osteogenic Differentiation of Human Mesenchymal Stem Cells, ACS Nano 5(6) (2011) 4670-4678.

[52] A.B. Seabra, A.J. Paula, R. de Lima, O.L. Alves, N. Durán, Nanotoxicity of Graphene and Graphene Oxide, Chem. Res. Toxicol. 27(2) (2014) 159-168.

[53] C. Bussy, H. Ali-Boucetta, K. Kostarelos, Safety Considerations for Graphene: Lessons Learnt from Carbon Nanotubes, Acc. Chem. Res. 46(3) (2013) 692-701.

[54] L. Chen, Z. Xu, J. Li, C. Min, L. Liu, X. Song, G. Chen, X. Meng, Reduction and disorder in graphene oxide induced by electron-beam irradiation, Mater. Lett. 65(8) (2011) 1229-1230.

[55] L. Tao, C. Qiu, F. Yu, H. Yang, M. Chen, G. Wang, L. Sun, Modification on Single-Layer Graphene Induced by Low-Energy Electron-Beam Irradiation, J. Phys. Chem. C 117(19) (2013) 10079-10085. 
[56] D. Teweldebrhan, A.A. Balandin, Modification of graphene properties due to electron-beam irradiation, Appl. Phys. Lett. 94(1) (2009).

[57] T.D. Yuzvinsky, A.M. Fennimore, W. Mickelson, C. Esquivias, A. Zettl, Precision cutting of nanotubes with a low-energy electron beam, Appl. Phys. Lett. 86(5) (2005) 1-3.

[58] D. Drouin, A.R. Couture, D. Joly, X. Tastet, V. Aimez, R. Gauvin, CASINO V2.42 - A Fast and Easy-to-use Modeling Tool for Scanning Electron Microscopy and Microanalysis Users, Scanning 29(3) (2007) 92-101.

[59] K.-H. Wu, H.-H. Cheng, A.A. Mohammad, I. Blakey, K. Jack, I.R. Gentle, D.-W. Wang, Electron-beam writing of deoxygenated micro-patterns on graphene oxide film, Carbon 95(Supplement C) (2015) 738-745.

[60] R.H. Telling, C.P. Ewels, A.A. El-Barbary, M.I. Heggie, Wigner defects bridge the graphite gap, 2 (2003) 333.

[61] P.V. Kumar, M. Bernardi, J.C. Grossman, The Impact of Functionalization on the Stability, Work Function, and Photoluminescence of Reduced Graphene Oxide, ACS Nano 7(2) (2013) 1638-1645.

[62] Y. Wang, Z. Qin, M.J. Buehler, Z. Xu, Intercalated water layers promote thermal dissipation at bio-nano interfaces, Nat. Commun. 7 (2016).

[63] M.J. McAllister, J.-L. Li, D.H. Adamson, H.C. Schniepp, A.A. Abdala, J. Liu, M. Herrera-Alonso, D.L. Milius, R. Car, R.K. Prud'homme, I.A. Aksay, Single Sheet Functionalized Graphene by Oxidation and Thermal Expansion of Graphite, Chem. Mater. 19(18) (2007) 4396-4404. 
[64] X. Chen, W. Li, D. Luo, M. Huang, X. Wu, Y. Huang, S.H. Lee, X. Chen, R.S. Ruoff, Controlling the Thickness of Thermally Expanded Films of Graphene Oxide, ACS Nano 11(1) (2017) 665-674.

[65] C. Mattevi, G. Eda, S. Agnoli, S. Miller, K.A. Mkhoyan, O. Celik, D. Mastrogiovanni, G. Granozzi, E. Garfunkel, M. Chhowalla, Evolution of Electrical, Chemical, and Structural Properties of Transparent and Conducting Chemically Derived Graphene Thin Films, Adv. Funct. Mater. 19(16) (2009) 2577-2583.

[66] P. Sun, Y. Wang, H. Liu, K. Wang, D. Wu, Z. Xu, H. Zhu, Structure evolution of graphene oxide during thermally driven phase transformation: Is the oxygen content really preserved?, PLoS ONE 9(11) (2014).

[67] D. Yang, A. Velamakanni, G. Bozoklu, S. Park, M. Stoller, R.D. Piner, S. Stankovich, I. Jung, D.A. Field, C.A. Ventrice Jr, R.S. Ruoff, Chemical analysis of graphene oxide films after heat and chemical treatments by X-ray photoelectron and Micro-Raman spectroscopy, Carbon 47(1) (2009) 145-152.

[68] S. Zhou, A. Bongiorno, Origin of the chemical and kinetic stability of graphene oxide, Sci. Rep. 3 (2013).

[69] S. Claramunt, A. Varea, D. López-Díaz, M.M. Velázquez, A. Cornet, A. Cirera, The Importance of Interbands on the Interpretation of the Raman Spectrum of Graphene Oxide, The J. Phys. Chem. C 119(18) (2015) 10123-10129.

[70] S. Eigler, C. Dotzer, A. Hirsch, Visualization of defect densities in reduced graphene oxide, Carbon 50(10) (2012) 3666-3673.

[71] G. Eda, M. Chhowalla, Chemically derived graphene oxide: Towards large-area thin-film electronics and optoelectronics, Adv. Mater. 22(22) (2010) 2392-2415. 
[72] W.C. Oliver, G.M. Pharr, An improved technique for determining hardness and elastic-modulus using load and displacement sensing indentation experiments, J. Mater. Res. 7(6) (1992) 1564-1583.

[73] Y. Liu, B. Xie, Z. Zhang, Q. Zheng, Z. Xu, Mechanical properties of graphene papers, Journal of the Mechanics and Physics of Solids 60(4) (2012) 591-605.

[74] A.R. Ranjbartoreh, B. Wang, X. Shen, G. Wang, Advanced mechanical properties of graphene paper, J. Appl. Phys. 109(1) (2011).

[75] M.C. Serrano, R. Pagani, M. Vallet-Regí, J. Peña, A. Rámila, I. Izquierdo, M.T. Portolés, In vitro biocompatibility assessment of poly( $\varepsilon$-caprolactone) films using L929 mouse fibroblasts, Biomaterials 25(25) (2004) 5603-5611.

[76] H. Ji, H. Sun, X. Qu, Antibacterial applications of graphene-based nanomaterials: Recent achievements and challenges, Adv. Drug Deliver. Rev. 105(Pt B) (2016) 176-189.

[77] R.J. Peláez, A. González-Mayorga, M.C. Gutiérrez, C. García-Rama, C.N. Afonso, M.C. Serrano, Tailored Fringed Platforms Produced by Laser Interference for Aligned Neural Cell Growth, Macromol. Biosci. 16(2) (2016) 255-265.

[78] L.C.Y. Lee, N. Gadegaard, M.C. de Andrés, L.-A. Turner, K.V. Burgess, S.J. Yarwood, J. Wells, M. Salmeron-Sanchez, D. Meek, R.O.C. Oreffo, M.J. Dalby, Nanotopography controls cell cycle changes involved with skeletal stem cell selfrenewal and multipotency, Biomaterials 116 (2017) 10-20. 


\section{Reductive nanometric patterning of graphene oxide paper using electron beam lithography}

Gil Gonçalves ${ }^{a *}$, Jérôme Borme ${ }^{b}$, Igor Bdkin ${ }^{a}$, Ankor González-Mayorga ${ }^{c}$, Gonzalo Irurueta $^{a}$, Helena I. S. Nogueira ${ }^{d}$ María C. Serrano $^{e, f, g}$, Pedro Alpuim ${ }^{b, e}$ Paula A.A.P. Marques $^{a^{*}}$

a TEMA-NRD, Mechanical Engineering Department, University of Aveiro, 3810-193 Aveiro, Portugal

${ }^{\mathrm{b}}$ INL - International Iberian Nanotechnology Laboratory, 4715-330, Braga, Portugal

${ }^{c}$ Hospital Nacional de Parapléjicos (HNP), Servicio de Salud de Castilla-La Mancha (SESCAM), Finca La Peraleda s/n, 45071-Toledo, Spain

${ }^{\mathrm{d}}$ CICECO, Department of Chemistry, University of Aveiro, 3810-193 Aveiro, Portugal

e Instituto de Ciencia de Materiales de Madrid (ICMM), Consejo Superior de Investigaciones Científicas (CSIC), 28049-Madrid, Spain

${ }^{\mathrm{f}}$ Materials Science Factory, ICMM-CSIC, 28049-Madrid, Spain

g Joint Research Unit "Design and Development of Biomaterials for Neural Regeneration", HNP-SESCAM, Joint Research Unit with CSIC

${ }^{\mathrm{h}}$ CFUM-Center of Physics of the University of Minho, 4710-057, Braga, Portugal

*Corresponding authors.

E-mail: ggoncalves@ua.pt (Gil Goncalves);E-mail: paulam@ua.pt (Paula Marques) 


\section{ACCEPTED MANUSCRIPT}

\section{List of Figures}

a)
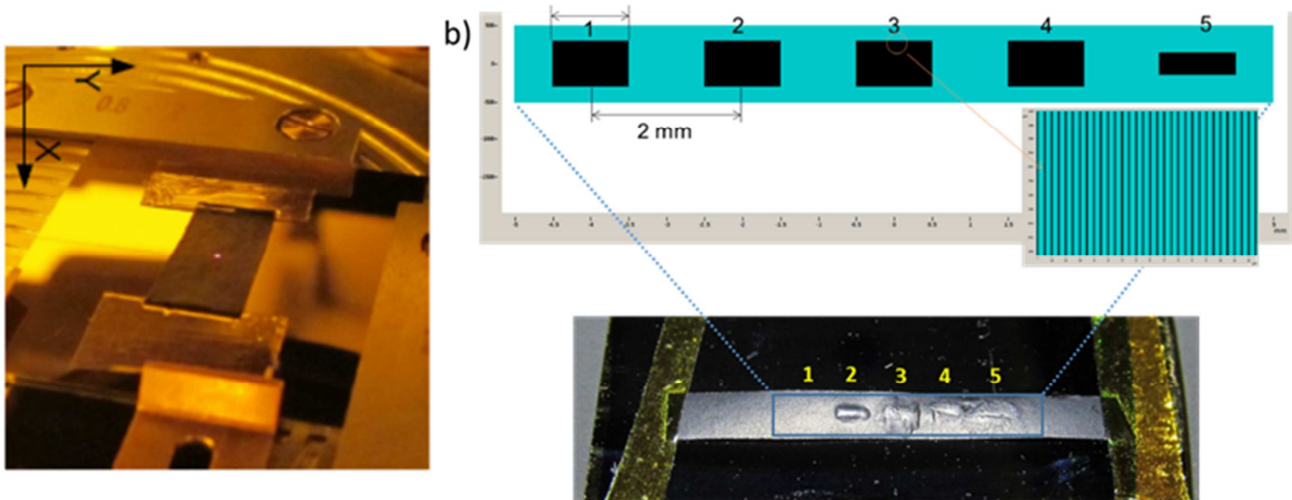

c)
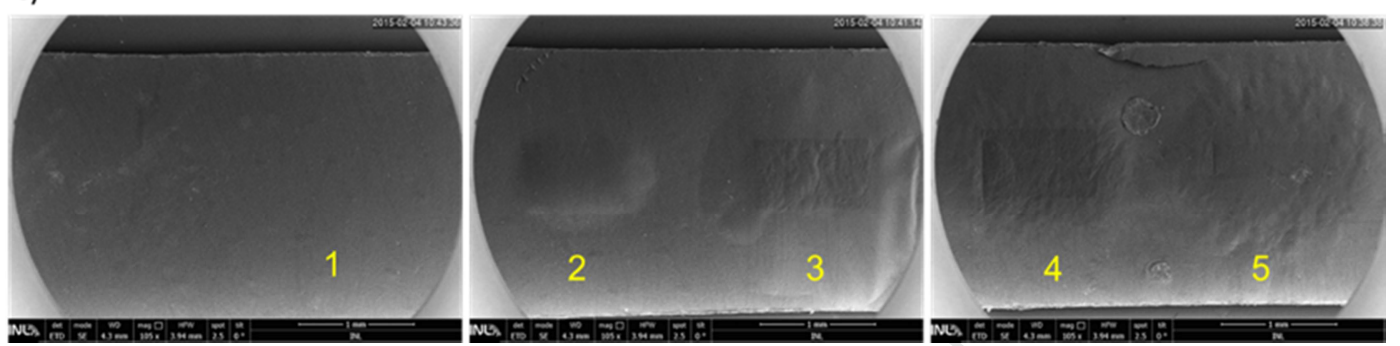

Figure 1. a) Photograph of a detail of the EBL apparatus used for the surface reduction patterning of GO paper. b) Schematic representation and optical photograph of the five exposed areas in a strip of the GO paper sample. c) Low-magnification SEM micrographs of the exposed samples, showing morphological changes in and around the rectangular exposed area, for the two largest doses used (4 and 5).
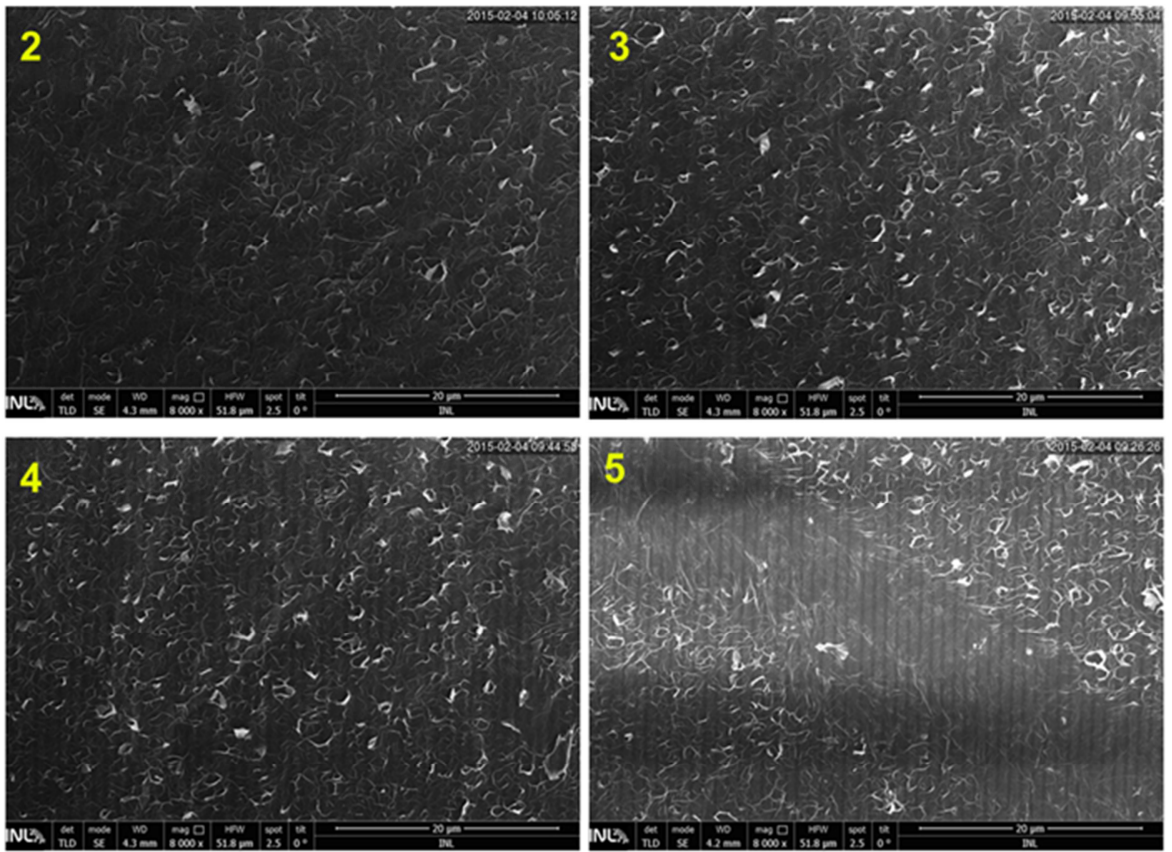
Figure 2. High magnification SEM micrographs of the areas exposed to different doses (2, 3, 4 and 5). For depositions at conditions 4 and 5, with the highest dose, a stripe pattern is visible.

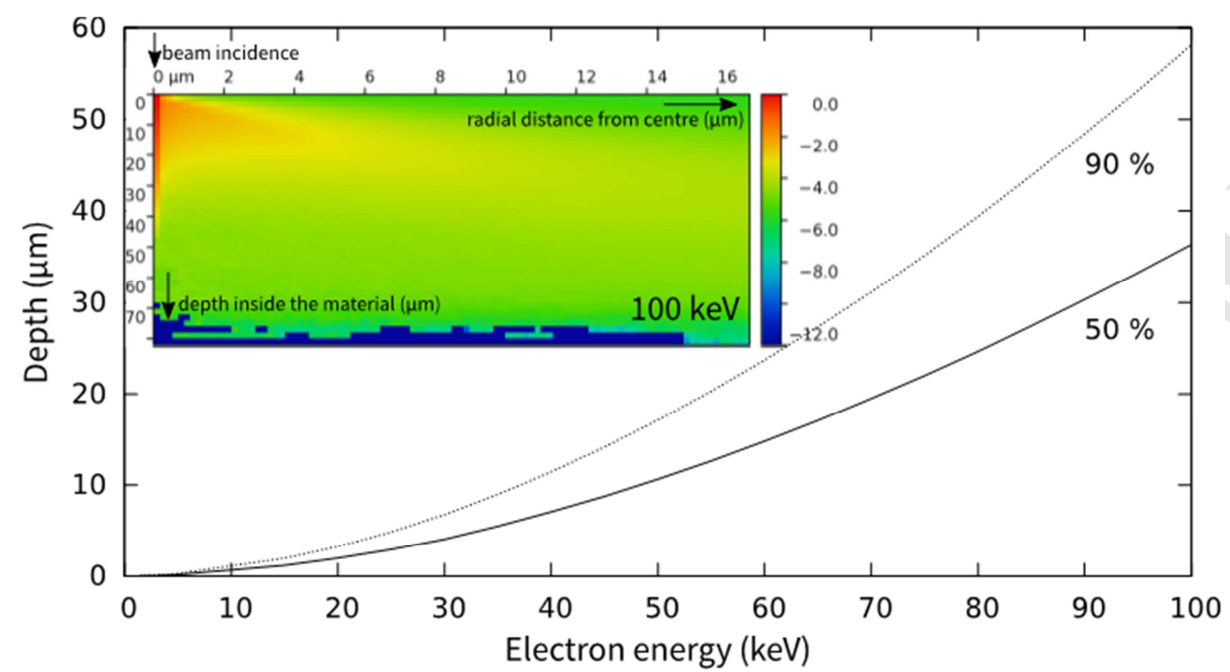

Figure 3. Depth of penetration of energy transferred by the electrons onto a GO material modelled as a material containing carbon and oxygen with $\mathrm{C} / \mathrm{O}=2.7$, simulated with Casino 2.48 [48], as function of the acceleration voltage of the incident electrons. In inset, the cross-sectional distribution of deposited energy at $100 \mathrm{kV}$, plotted as a radial distribution. The arrow at $(0,0)$ in the inset indicates the place of beam incidence. The colour scale corresponds to the decimal logarithm of energy distribution. 
a)
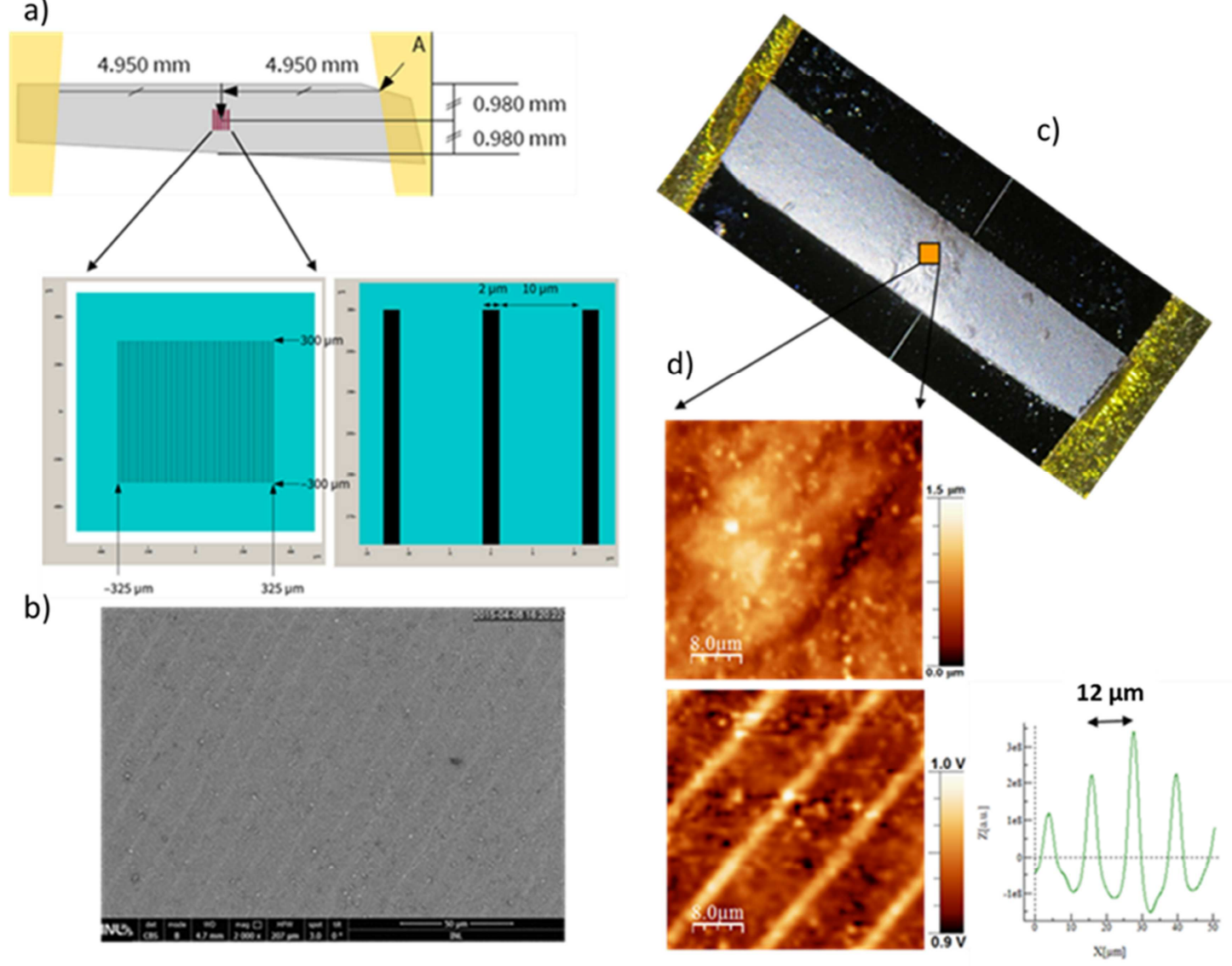

Figure 4. a) Schematic representation of the patterned performed on the surface of the GO paper by EBL. b) SEM image of patterned GO paper. c) Optical photograph of the exposed area on a strip of the GO paper sample. d) AFM measurements of the surface potential of the patterned GO paper, showing the predefined center-to-center distance between the reduced lines of $12 \mu \mathrm{m}$.
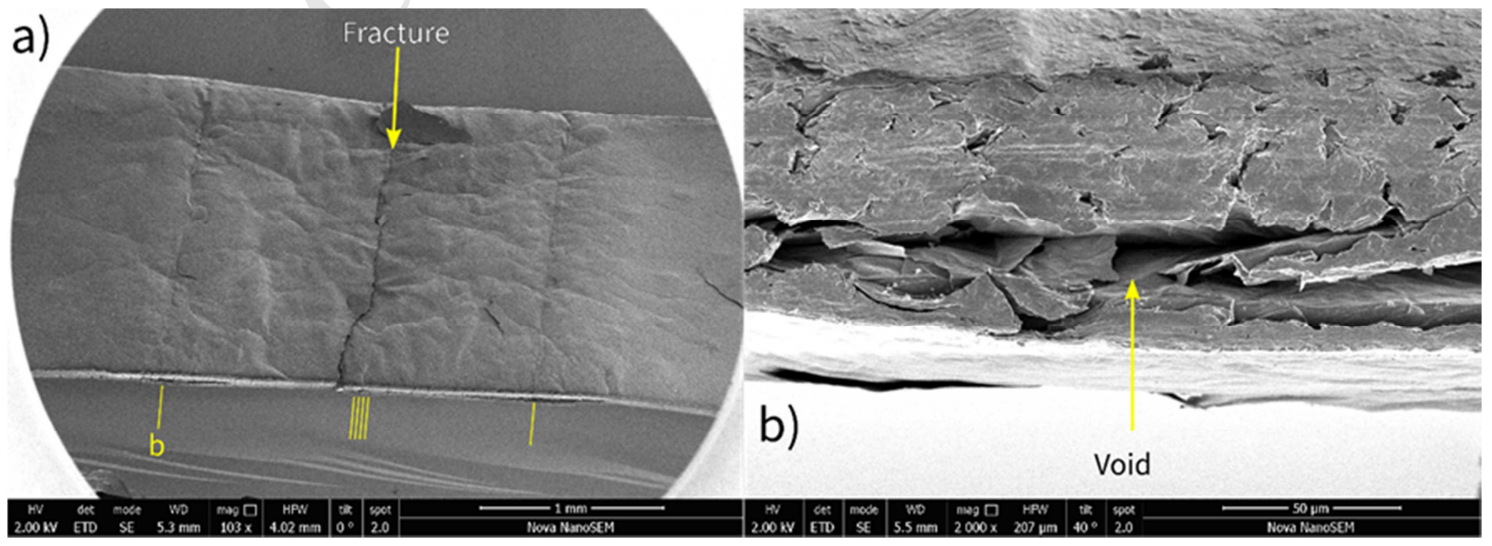
Figure 5. SEM observation of a sample patterned using the $100 \mathrm{kV}$ electron beam in the shape of lines crossing its entire width. a) Low magnification image under a $45^{\circ}$ tilt. The marked lines denote the places of exposure: one central region with eight exposed lines, and two single lines on either side of the central region. The central area exhibits a continuous fracture across the sample, which appeared during the manipulation of the sample. b) Close-up on the area marked b in image a), under a tilt of $85^{\circ}$. This image shows the structure in sheets of GO and the voids caused by reduction, following the exposure. It also allows evaluation of the thickness of the GO paper in the area exposed to the electron beam.

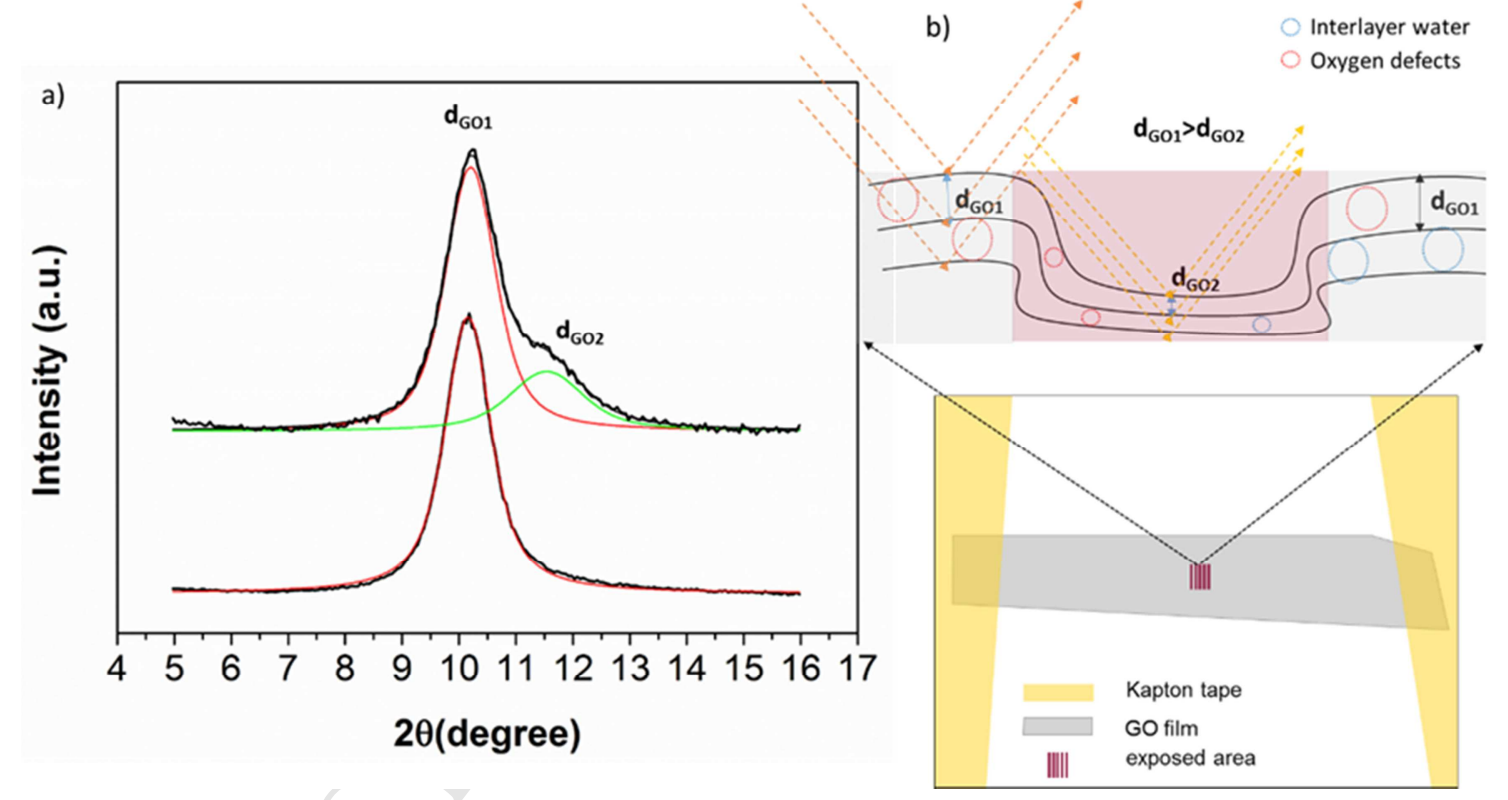

Figure 6. a) XRD of GO paper performed on the patterned and non-patterned regions by EBL. b) Schematic representation of the interlayer distance of GO sheets on the paper after electron beam exposure. 

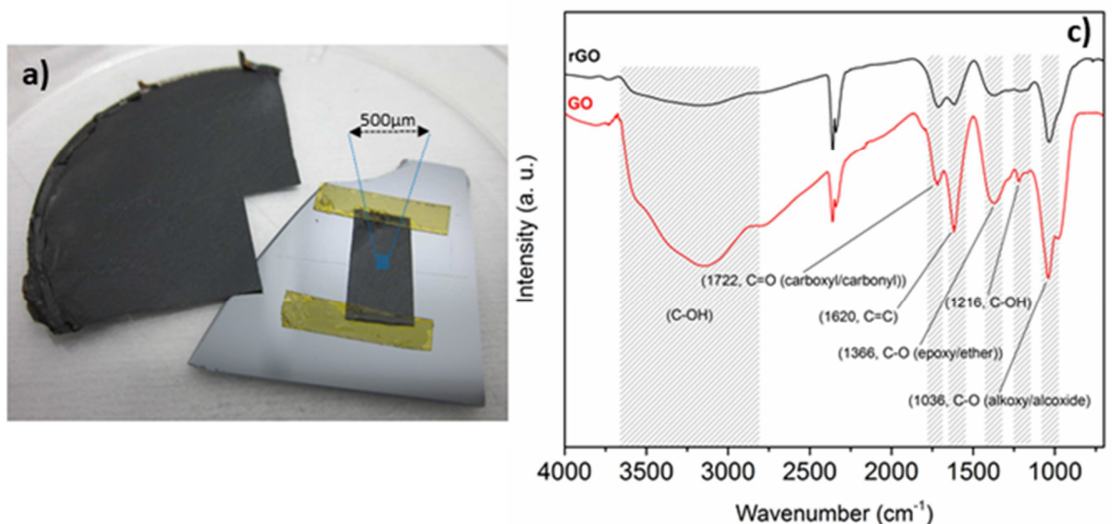

b)
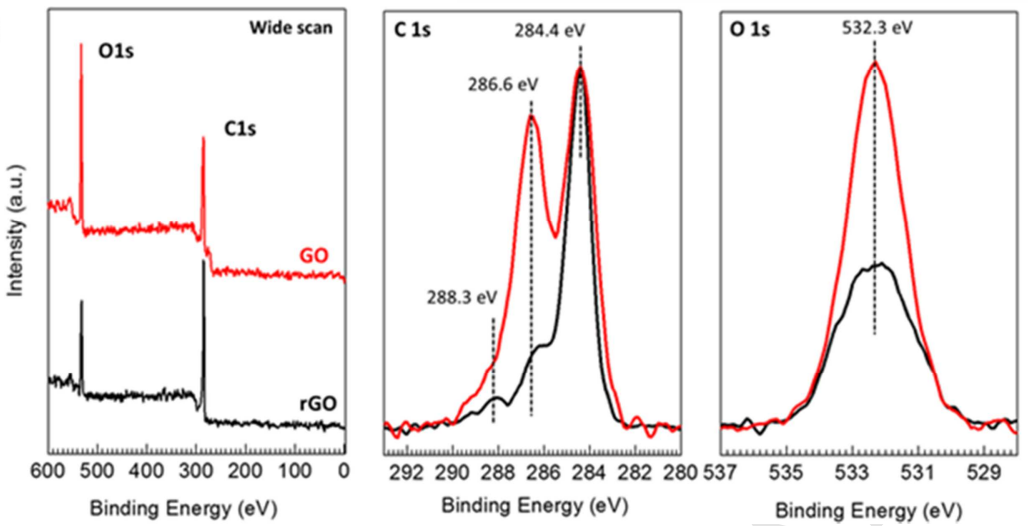

Figure 7. a) GO papers with patterned area of $500 \mu \mathrm{m}^{2}$ by EBL (blue region). b) XPS and c) FTIR spectra of the GO paper on the patterned region (rGO) and outside of the patterned region $(\mathrm{GO})$. 

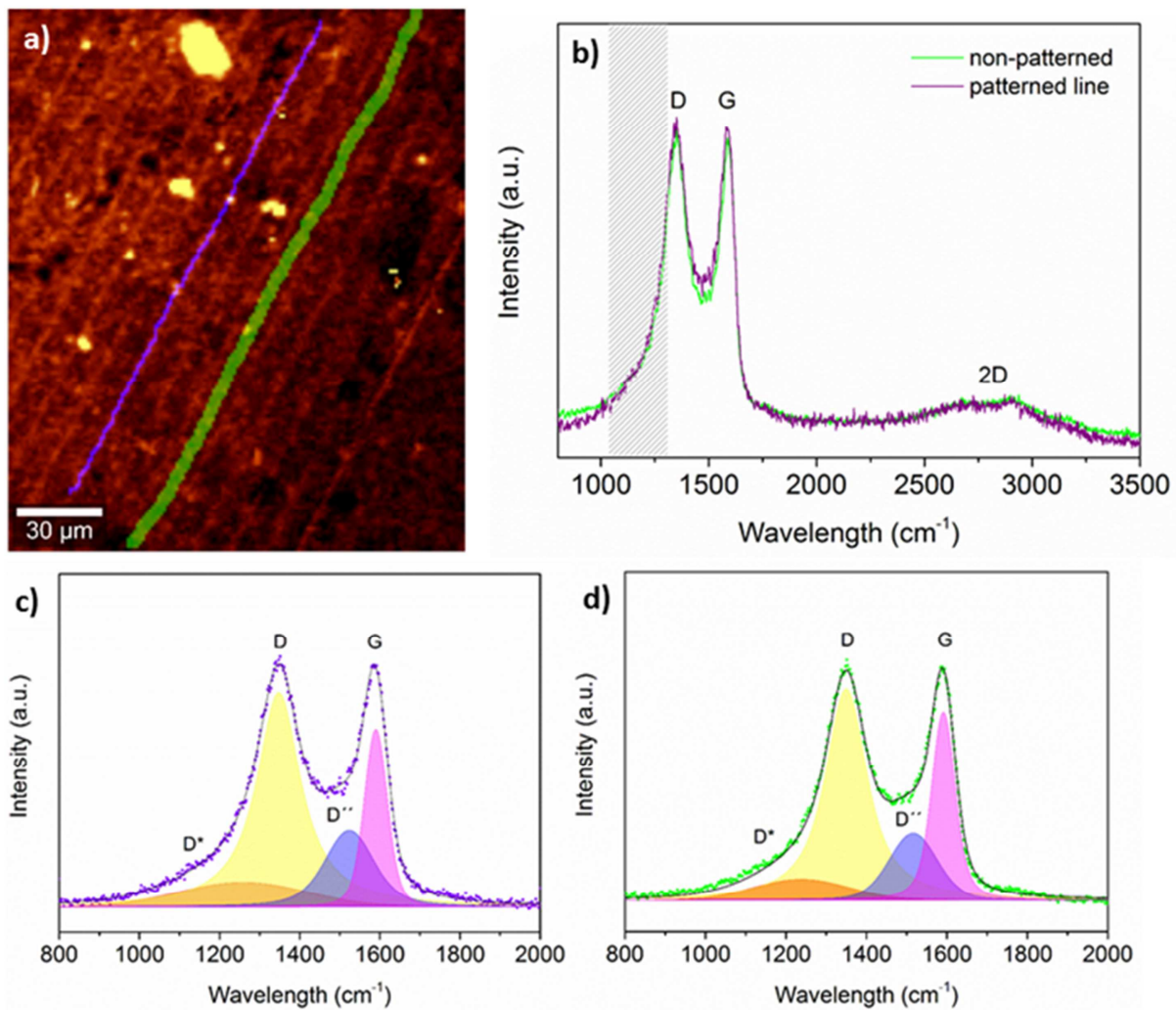

Figure 8. a) Raman image of the patterned region of the GO paper, obtained using the integrated intensity of the dashed region in $\mathrm{b}\left(\sim 1100-1300 \mathrm{~cm}^{-1}\right)$; areas with reduced (patterned) and non-reduced GO (non-patterned) were marked by the violet and the green lines, respectively. b) Raman spectra obtained by the medium spectra of all pixels in the respective selected areas in (a), violet for patterned GO and green for nonpatterned GO. Fitting of the D and G peaks is shown in c) for patterned GO and in d) for non-patterned GO. 
a)

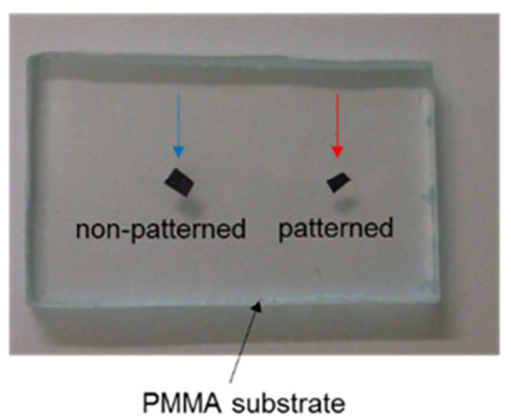

c)

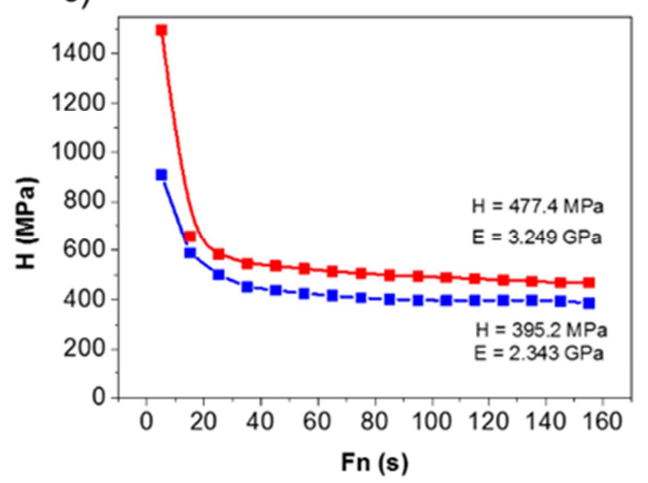

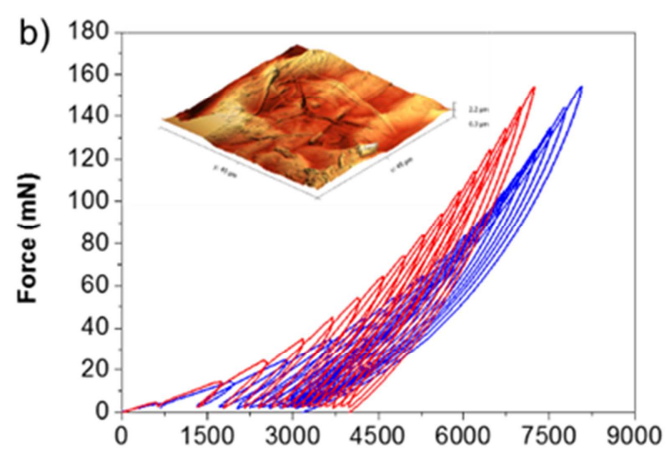

d)

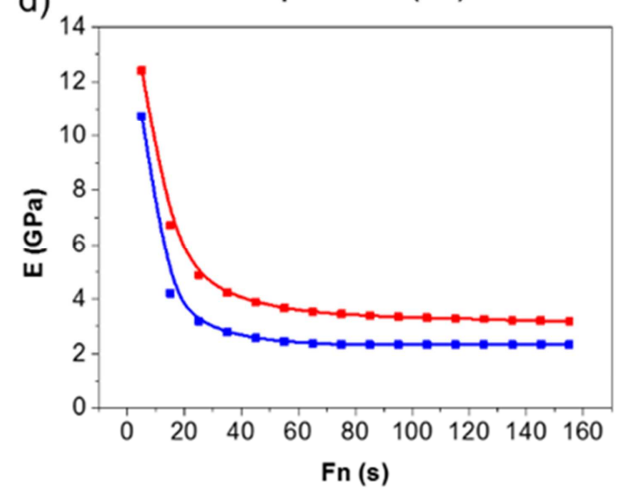

Figure 9. Representative load-displacement curves obtained for the GO papers on the patterned and non-patterned regions by EBL.
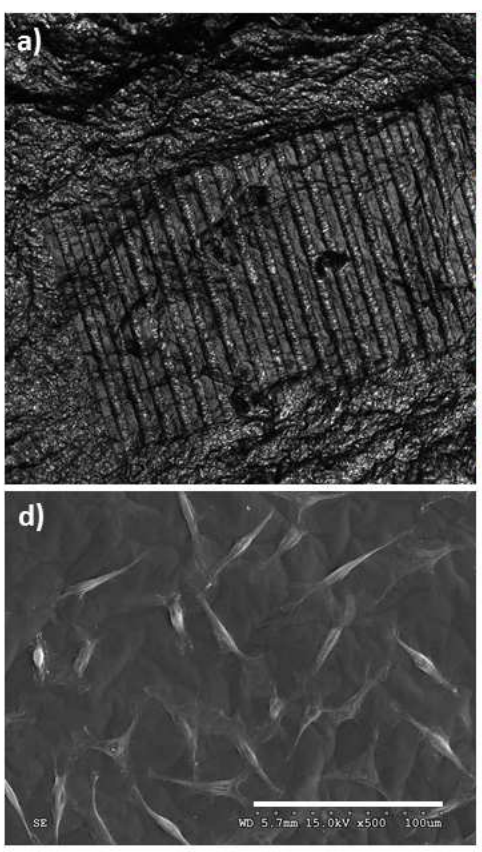
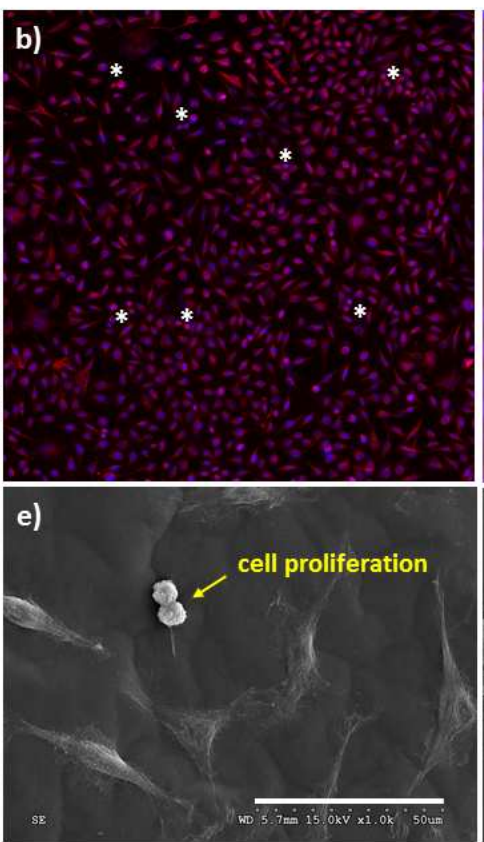

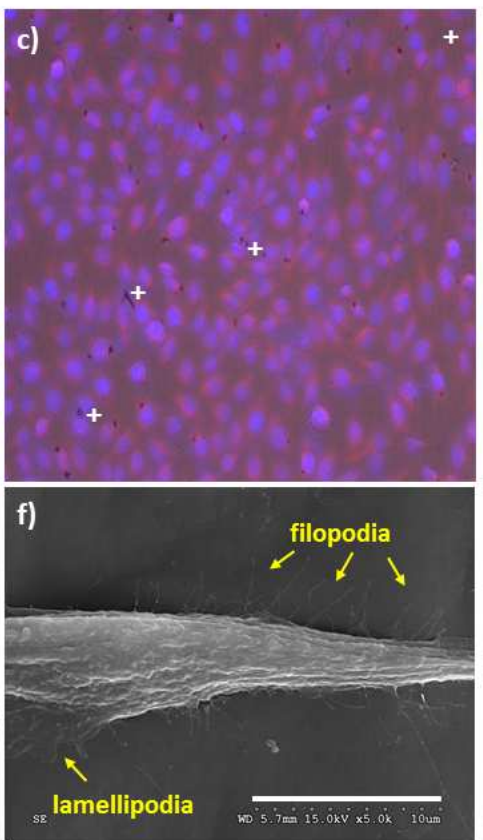

Figure 10. Cytocompatibility in vitro studies of L929 fibroblasts cultured on GO paper.

CLSM micrograph in reflexion mode illustrating the pattern position in the film a). 
Adhesion and cytoskeletal visualization by CLSM on both patterned and non-patterned areas b) and at the periphery of the GO paper on polystyrene (c). Cells in active proliferation are labelled with white asterisks and GO film fragments with white crosses. Scale bars: $150 \mu \mathrm{m}(\mathrm{a}, \mathrm{b})$ and $50 \mu \mathrm{m}$ c). Morphological studies by SEM (d-f). Cells undergoing cytokinesis and detailed cytoplasmic projections are indicated by yellow arrows. Scale bars: 100, 50 and $10 \mu \mathrm{m}$ (d, e and f, respectively). 


\section{List of tables}

Table 1. Experimental conditions tested for the patterning of the GO paper by EBL.

\begin{tabular}{llllll}
\hline Exposure & $\mathbf{1}$ & $\mathbf{2}$ & $\mathbf{3}$ & $\mathbf{4}$ & $\mathbf{5}$ \\
\hline Dose $\left(\times 10^{3} \mu \mathrm{C} / \mathrm{cm}^{2}\right)$ & 0.8 & 8 & 80 & 800 & 8000 \\
Line length $(\mu \mathrm{m})$ & 600 & 600 & 600 & 600 & $300\left(^{*}\right)$ \\
Step size $(\mathrm{nm})$ & 25 & 20 & 20 & 20 & 20 \\
Dwell time $(\mathrm{ns})$ & 30 & 192 & 1919 & 19190 & 191904 \\
Beam linear velocity $(\mu \mathrm{m} / \mathrm{s})$ & 834 & 104 & 10.4 & 1.04 & 0.104 \\
& 53.73 & 104.78 & 617.01 & 5792.34 & 28776.55 \\
Exposure duration $(\mathrm{s})$ & $\approx 1 \mathrm{~min}$ & $\approx 1 \mathrm{~min} 45$ & $\approx 10 \mathrm{~min}$ & $\approx 1 \mathrm{~h} 35$ & $\approx 8 \mathrm{~h}$ \\
& & & & & \\
\hline
\end{tabular}

$\left(^{*}\right)$ half the length to reduce exposure time to reasonable value

Table 2. Quantitative data obtained from the XPS spectra for the patterned and nonpatterned regions of GO paper.

\begin{tabular}{|c|c|c|c|c|c|c|}
\hline & & C1s fit & & & & \\
\hline Sample region & Component & $\mathrm{BE}(\mathrm{eV})$ & FWHM (eV) & Functional group & $\%$ & \\
\hline \multirow{3}{*}{ patterned } & 1 & 284.4 & 1.1 & $\mathrm{C} \mathrm{Sp}^{2}$ & 68 & \multirow{3}{*}{4.3} \\
\hline & 2 & 286.1 & 1.8 & $\mathrm{C}-\mathrm{O}$ & 24 & \\
\hline & 3 & 288.4 & 2.1 & $\mathrm{C}=\mathrm{O}$ & 8 & \\
\hline \multirow{3}{*}{ Non-patterned } & 1 & 284.4 & 1.5 & $\mathrm{C} \mathrm{Sp}^{2}$ & 48 & \multirow{3}{*}{2.7} \\
\hline & 2 & 286.5 & 1.6 & $\mathrm{C}-\mathrm{O}$ & 40 & \\
\hline & 3 & 288.0 & 2.5 & $\mathrm{C}=\mathrm{O}$ & 12 & \\
\hline
\end{tabular}


Table 3. Resume of the structural properties of GO patterned paper by ELB in comparison to non-modified GO paper

\begin{tabular}{|c|c|c|c|c|c|}
\hline \multirow{2}{*}{$\begin{array}{l}\text { GO patterned } \\
\text { paper }\end{array}$} & \multicolumn{5}{|c|}{ Structural characterization } \\
\hline & XRD & Raman & XPS & Resistivity & Nanoindentation \\
\hline non-patterned region & $\begin{array}{l}2 \theta=10.15^{\circ} \\
(\mathrm{d} \sim 8.7 \AA)\end{array}$ & $\mathrm{D}^{*}{ }_{\max }=1238 \mathrm{~cm}^{-1}$ & $\mathrm{C} 1 \mathrm{~s} / \mathrm{O} 1 \mathrm{~s}$ ratio $=4.3$ & $14 \mathrm{~K} \Omega \mathrm{m}$ & $\begin{aligned} \mathrm{H} & =395.2 \mathrm{MPa} \\
\mathrm{E} & =2.343 \mathrm{GPa}\end{aligned}$ \\
\hline patterned region* & $\begin{array}{c}2 \theta=10.20^{\circ} \\
(\mathrm{d} \sim 8.7 \AA) \\
2 \theta=11.53^{\circ} \\
(\mathrm{d} \sim 8.7 \AA)\end{array}$ & $\mathrm{D}^{*}{ }_{\max }=1258 \mathrm{~cm}^{-1}$ & $\mathrm{C} 1 \mathrm{~s} / \mathrm{O} 1 \mathrm{~s}$ ratio $=2.7$ & $0.44 \Omega \mathrm{m}$ & $\begin{array}{l}\mathrm{H}=477.4 \mathrm{MPa} \\
\mathrm{E}=3.249 \mathrm{GPa}\end{array}$ \\
\hline
\end{tabular}

*exposure ELB experimental conditions number 5 reported on table 1 